\title{
Neuron
}

\section{A Cellular-Resolution Atlas of the Larval Zebrafish Brain}

\section{Graphical Abstract}

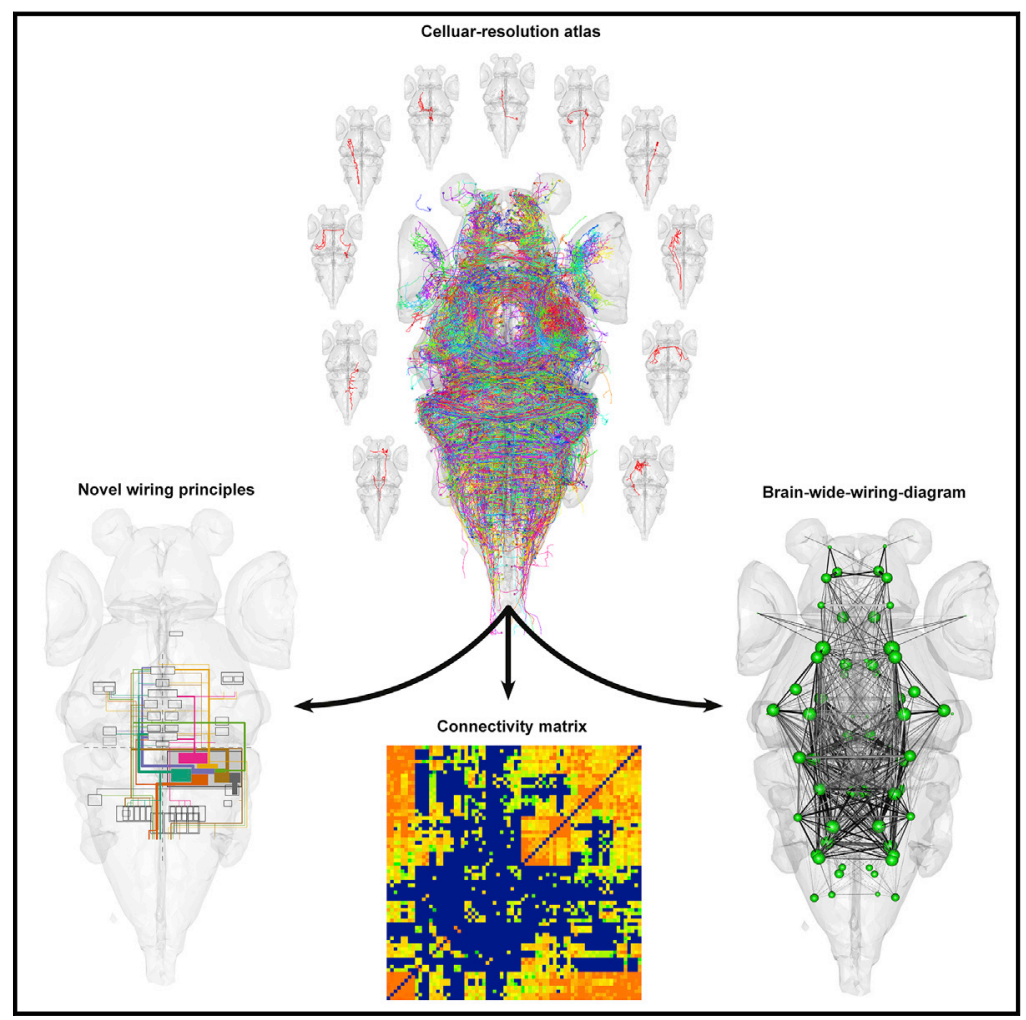

Highlights

- Generation of an interactive database with over 2,000 zebrafish neurons

- Discovery of novel wiring principles for tectum and cerebellum

- Hierarchical clustering based on similarity revealed novel neuronal morphotypes

- First brain-wide inter-areal wiring diagram with cellular resolution in a vertebrate

\section{Authors}

Michael Kunst, Eva Laurell, Nouwar Mokayes, ...,

Dominique Förster, Marco Dal Maschio, Herwig Baier

\section{Correspondence}

hbaier@neuro.mpg.de

\section{In Brief}

Kunst et al. generated a digital atlas of the larval zebrafish brain containing over 2,000 neuron morphologies, most of them previously unknown. These data are used to generate the first brain-wide wiring diagram with single-cell resolution for a vertebrate species. 


\title{
A Cellular-Resolution Atlas of the Larval Zebrafish Brain
}

\author{
Michael Kunst, ${ }^{1}$ Eva Laurell, ${ }^{1}$ Nouwar Mokayes, ${ }^{1}$ Anna Kramer, ${ }^{1}$ Fumi Kubo,, ${ }^{1,2}$ António M. Fernandes, ${ }^{1}$ \\ Dominique Förster, ${ }^{1}$ Marco Dal Maschio, ${ }^{1,3}$ and Herwig Baier ${ }^{1,4, *}$ \\ ${ }^{1}$ Department Genes - Circuits - Behavior, Max Planck Institute of Neurobiology, 82152 Martinsried, Germany \\ 2Present address: National Institute of Genetics, Center for Frontier Research, 1111 Yata, Mishima, Shizuoka 411-8540, Japan \\ ${ }^{3}$ Present address: Department of Biomedical Sciences, Padua Neuroscience Center, University of Padua, 35158 Padua, Italy \\ ${ }^{4}$ Lead Contact \\ ${ }^{*}$ Correspondence: hbaier@neuro.mpg.de \\ https://doi.org/10.1016/j.neuron.2019.04.034
}

\section{SUMMARY}

Understanding brain-wide neuronal dynamics requires a detailed map of the underlying circuit architecture. We built an interactive cellular-resolution atlas of the zebrafish brain at 6 days post-fertilization (dpf) based on the reconstructions of over 2,000 individually GFP-labeled neurons. We clustered our dataset in "morphotypes," establishing a unique database of quantitatively described neuronal morphologies together with their spatial coordinates in vivo. Over 100 transgene expression patterns were imaged separately and co-registered with the single-neuron atlas. By annotating 72 non-overlapping brain regions, we generated from our dataset an inter-areal wiring diagram of the larval brain, which serves as ground truth for synapse-scale, electron microscopic reconstructions. Interrogating our atlas by "virtual tract tracing" has already revealed previously unknown wiring principles in the tectum and the cerebellum. In conclusion, we present here an evolving computational resource and visualization tool, which will be essential to map function to structure in a vertebrate brain.

\section{INTRODUCTION}

The brain's ability to perceive sensory information and coordinate movement is based on the dynamic interactions of interconnected nerve cells, which are often distributed over several brain regions. Due to the complexity of neuronal networks, it is challenging to pinpoint the role of individual cells, or cell types, in these processes. Progress should come from efforts that successfully link patterns of neural activity to their anatomical substrate (Alivisatos et al., 2012; Bauer et al., 2018; Panzeri et al., 2017; Sporns et al., 2005). For functional neuroanatomy to be informative, connectivities among neurons have to be determined in a systematic, unbiased fashion and visualized in an atlas format. Resolving the entire wiring diagram of a brain re- quires dense electron-microscopic reconstructions of nervous tissue volumes, preferably at the scale of individual synapses (Denk and Horstmann, 2004; Hildebrand et al., 2017; Ohyama et al., 2015; Varshney et al., 2011; Zheng et al., 2018). To understand basic organizational features of the brain, however, synapse-scale resolution may often not be necessary. Rather, mesoscopic descriptions of brain connectivity using light microscopy (LM) provide a sufficient framework for the generation of testable hypotheses (Bohland et al., 2009; Mitra, 2014). Furthermore, building electron microscopy (EM) connectomes relies on the tracing of fibers over hundreds or thousands of sections. A catalog of reproducible cell shapes and inter-areal projection patterns will serve as "ground truth" to weed out continuation and merging errors and thus speed up ongoing EM efforts.

A brain-wide wiring diagram based on single-neuron reconstructions has been generated for Drosophila melanogaster (Chiang et al., 2011), revealing the general network structure and predicting whole-brain information flow (Shih et al., 2015). A similar approach has recently been performed in mice (Oh et al., 2014). However, due to the large size and complexity of the mouse brain, it was not feasible to perform this approach at the level of individual cells; instead, a grid-based approach to map anatomical connections between brain regions was used. This has the disadvantage that multiple cell types are labeled and information about individual collateral branching patterns is lost (Mitra, 2014). Also, there are currently no methods available to measure whole-brain activity with cellular resolution in mice.

Larval zebrafish (Danio rerio) offer several advantages for a comprehensive brain atlas project. Their translucency and small brain size, combined with an extensive arsenal of genetic tools (Baier and Scott, 2009; Friedrich et al., 2013) as well as quantitative behavioral assays (Orger and de Polavieja, 2017), make it possible to monitor and manipulate brain-wide neural activity, while the animal is responding to sensory stimuli and displaying movements of the eyes and tail (Ahrens et al., 2013; Portugues et al., 2014; Dal Maschio et al., 2017). Here, we have expanded the larval zebrafish toolkit by creating a digital neuroanatomy platform, which includes an unbiased catalog of cell morphologies, together with their spatial coordinates and long-range projections.

Our approach employed the stochastic and unbiased labeling of individual neurons in different, but age-matched, brains, which 
are then morphed into a common coordinate system ("standard brain") (see Hawrylycz et al., 2011). We show here that this format is expandable, as it can easily incorporate other reconstructed neurons. Importantly, transgene expression patterns (e.g., from the growing collection of zebrafish Gal4, Cre, and Q lines) can be straightforwardly superimposed, instantly returning information about circuit composition and sharpening hypotheses about potential connectivity. To illustrate the power of this evolving resource, we have extracted new topographic principles in the tectum and cerebellum. Extending this approach to the whole brain, a first version of the connectome of the larval zebrafish brain has emerged. Computational models of circuit function are currently based solely on imaging with slow optical indicators and are therefore largely unconstrained by anatomical realities (Naumann et al., 2016). The open and interactive platform architecture of our new resource makes it possible to integrate optical recordings with cell-resolved anatomy of the activated brain regions. This application of our atlas resource has already been realized in the accompanying article by Kramer et al. (2019, this issue of Neuron).

\section{RESULTS}

\section{A New Digital Resource Allows Co-registration of} Independent Neuron Tracings to a Standard Brain To digitally catalog the morphologies and positions of neurons and compare them among individual fish, we developed a workflow to label, scan, and digitize single neurons and subsequently align them to a common coordinate framework (Figure 1A). To reveal the morphology of neurons, we made use of a fluorescent reporter transgene, BGUG, which encodes a highly variegated, membrane-targeted GFP driven from the upstream activating sequence (UAS) of the yeast transcription factor Gal4 (Xiao and Baier, 2007). When crossed to Gal4-VP16 driver lines, this approach leads to extremely sparse GFP expression in one, or very few, random neurons that are part of the overall Gal4 pattern. We used pan-neuronally expressing Gal4 lines (elav/3: Gal4 and Gal4s1101t) to sample neurons from all brain regions in an unbiased manner. We focused our attention on neurons that can be traced in their entirety, excluding reticulospinal projection neurons. To generate high signal-to-noise ratio, we adapted the passive CLARITY method (PACT) (Treweek et al., 2015) to larval zebrafish. As the clearing method quenches the GFP fluorescence, we visualized labeled neurons via antibody staining directed against GFP epitopes. Each brain was also stained with an antibody to Synapsin (SYP), which outlines the neuropil areas and yields a highly stereotyped pattern across individuals.

In order to co-register individual BGUG-labeled neurons, we first generated a standard brain by averaging the SYP patterns from 12 larval zebrafish at 6 days post-fertilization (dpf) (Figures $1 \mathrm{~B}$ and $\mathrm{C}$ ). To account for deformations introduced by our labeling protocol, we used the iterative symmetric group-wise normalization (SyGN) approach provided by the advanced normalization tool (ANT) toolkit (Avants et al., 2010). This iterative process does not favor any one individual but rather represents an unbiased average of the population (Figures $1 \mathrm{C}$ and S1). Individual fish brains containing BGUG-labeled cells were then aligned to this template using the SYP reference channel, and the transformation was applied computationally to the traced neurons (Figure 1D). Using this workflow, we generated an atlas of 1,709 stochastically chosen neurons that cover the entire brain.

To demonstrate that our labeling technique samples from the entire brain in an unbiased fashion, we analyzed the distribution of the somas of traced neurons in 36 brain regions (see below) and compared it to a mask of cell bodies (Figure S2A). When we compared the relative distribution, we could find that both soma location and cell body mask correlated well, i.e., regions with a relatively large cell body area also contained a higher number of somas from traced neurons $(R=0.8$, Figures S2B and $\mathrm{S} 2 \mathrm{C})$. In addition, since our single-cell labeling is based on the Gal4/UAS-expression system, we are also able to target certain cell types using more restrictive driver lines. To demonstrate this, more than two hundred tracings, obtained using more specific drivers (vglut2a:Gal4, Gal4s1171t, Ihx9:Gal4, Gal4s1013t), were added (Figure 1E; Video S1). When we analyzed this dataset for labeling bias, we found that the correlation increased with the added neurons $(R=0.59$, Figure S2D). This improved correlation was most notable in the case of the tectum (arrow in Figure S2E). Taken together, our workflow labels neurons throughout the brain in an apparently unbiased fashion, reflecting the expression pattern of the Gal4 driver used.

\section{Single-Neuron Morphologies Can Be Aligned to Transgene Expression Patterns and Other Data Modalities}

To provide flexibility for efforts using reference channels other than SYP, we generated shape-based averages for commonly used immunohistochemical labels and transgenic reporters (Figure $2 A)$. These include ERK1/2 and HuC/D antibodies, as well as the elavl3:IynTagRFP, bAct2B:GFP, vGlut2a:DsRed, elavl3: GCaMP5G, elav/3:n/sGCaMP6S, and elav/3:H2BGCaMP6S transgenic reporter lines. With the aid of these bridging registrations, we crosslinked our database to existing anatomical resources, including Z-Brain (Randlett et al., 2015) and the Zebrafish Brain Browser (Marquart et al., 2015). Importantly, we could also incorporate the expression patterns of Gal4 lines generated by various laboratories into our platform (Asakawa et al., 2008; Förster et al., 2017; Scott et al., 2007) (Figure 2B).

Rather than imaging the entire brain, researchers often prefer a more targeted approach, focusing on a specific brain region (e. g., Kubo et al., 2014). To accommodate such datasets in our resource, we developed a computational pipeline that enables us to register subvolumes to the standard brain. As a proof of principle, we aligned a set of 55 mitral cell tracings from a previous publication (Miyasaka et al., 2014). Mitral cells are projection neurons that carry information from the olfactory bulbs to several forebrain regions. In this case, only the brain subvolume containing the forebrain and habenula had been acquired using the presynaptic marker SV2 as the reference channel. Reasoning that SV2 should have a similar labeling pattern to SYP, we cropped our SYP reference stain to match the region imaged by Miyasaka et al. and used this as the template to successfully align their mitral cell tracings to our standard brain (Figure 2C). Finally, we integrated a large-scale dataset of tectal 
A

Sparse labeling

Fixation and clearing

Labeling and imaging
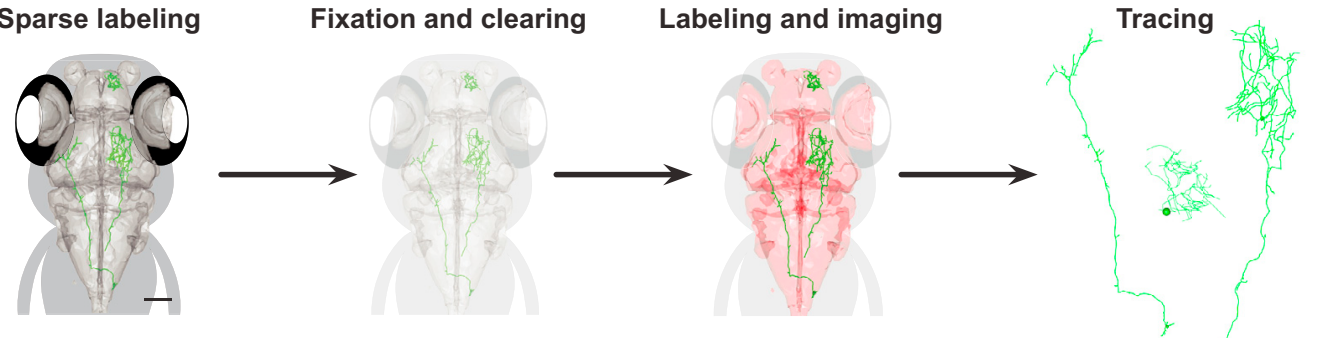

B
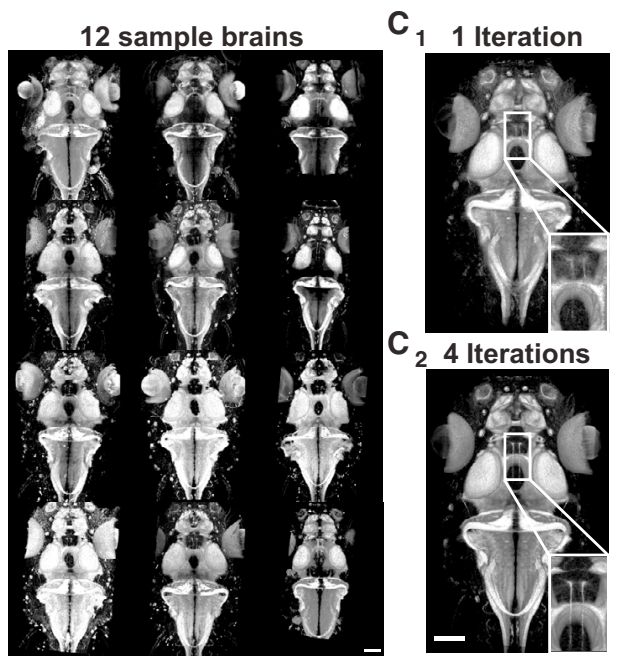

E

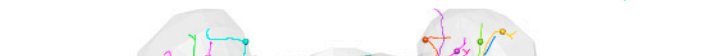

D
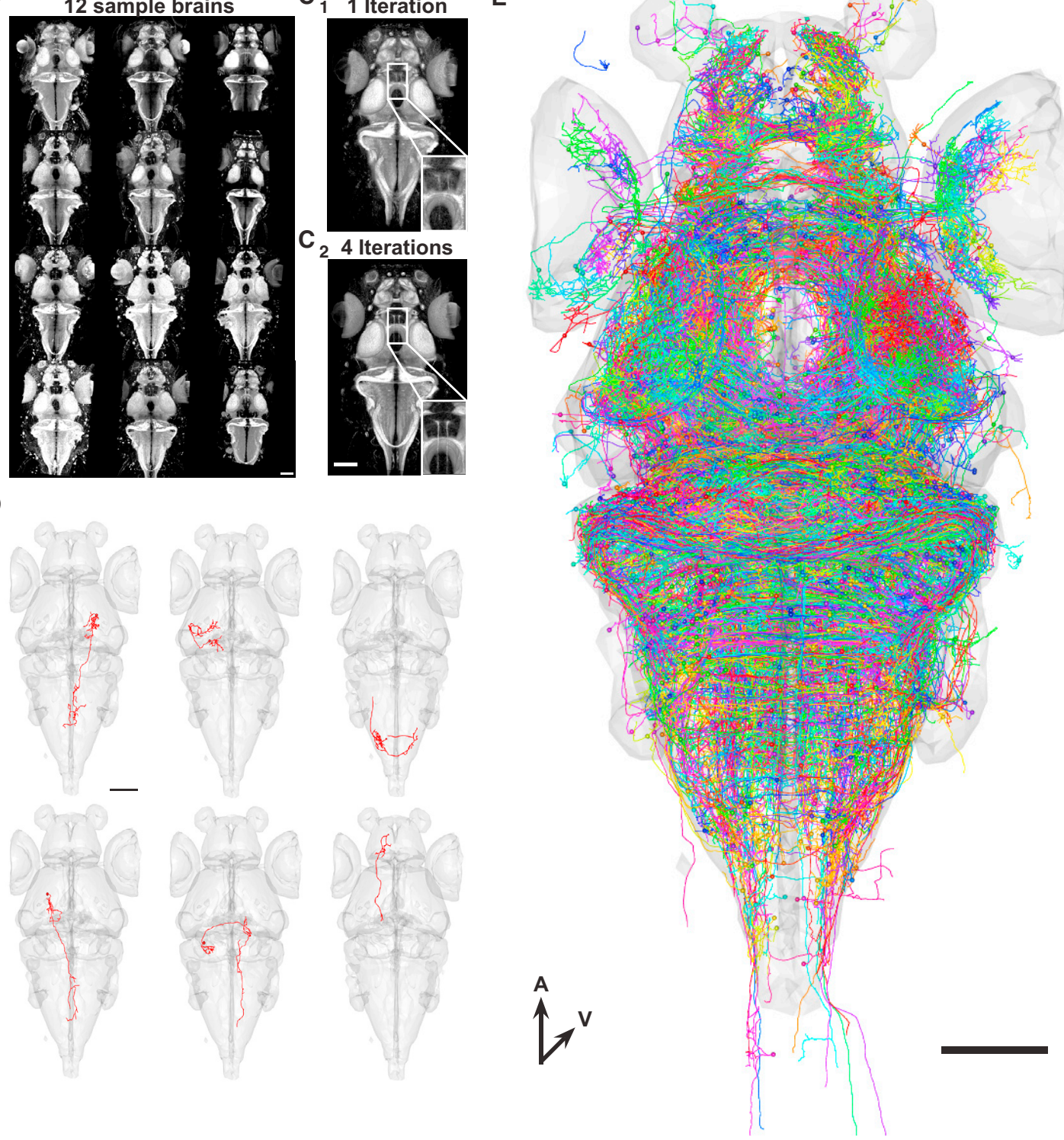

Figure 1. Construction of a Single-Neuron Atlas

(A) Workflow for generating single-neurons tracings.

(B) Images of 12 representative brains used to generate a shape-based average template. Brains were labeled with an antibody against the presynaptic protein Synapsin.

(C) Shape-based average template brain after 1 iteration $\left(C_{1}\right)$ and 4 iterations $\left(C_{2}\right)$. Inset shows higher magnification of the torus longitudinalis neuropil.

(D) Examples of individually traced neurons from different brains aligned to our template brain (gray surface).

(E) Overlay of all neurons $(n=1,955)$ generated by our workflow. Colors of neurons are assigned randomly.

All scale bars represent $100 \mu \mathrm{m}$. 
A

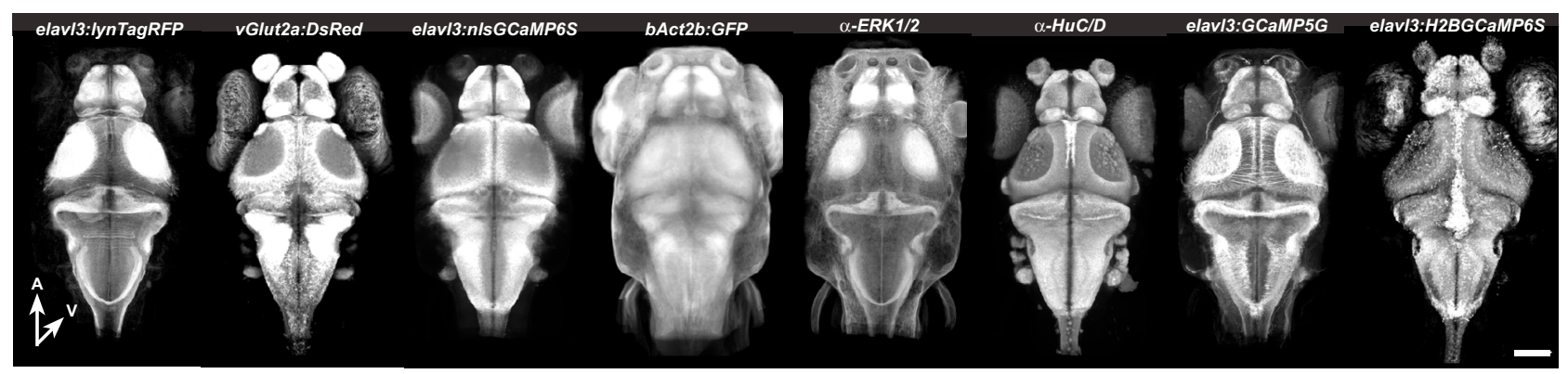

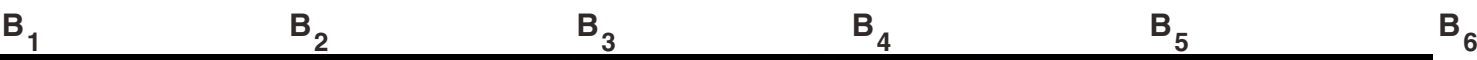

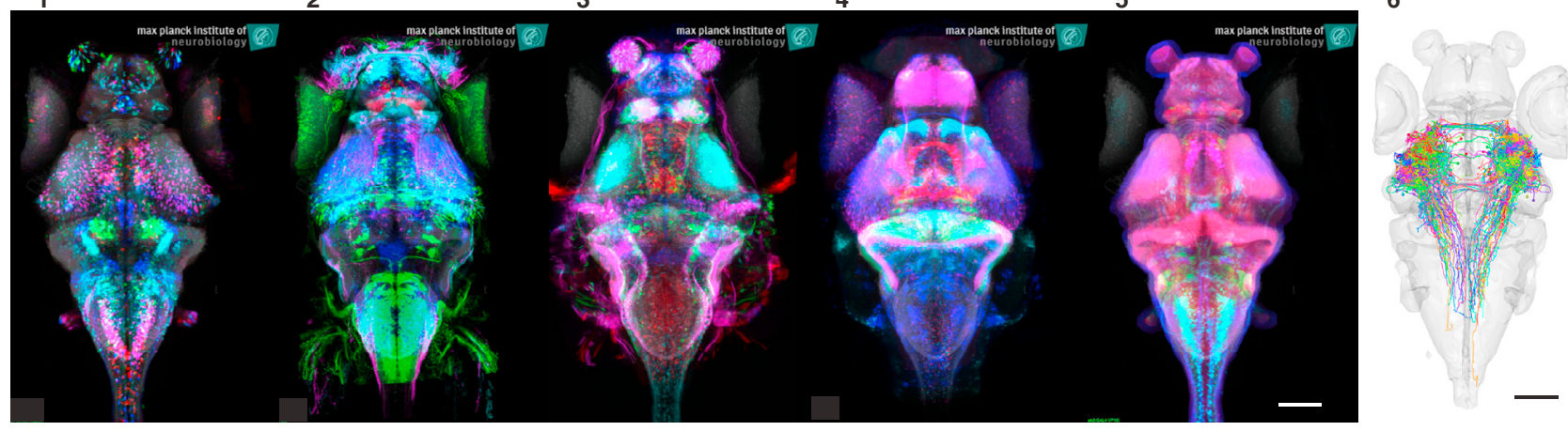

From Marquart et al., 2015

From Förster et al., 2017

From Scott et al., 2007

From Asakawa et al., 2007

From Randlett et al., 2015

C

Imaged subvolume from Miyasaka et al.,
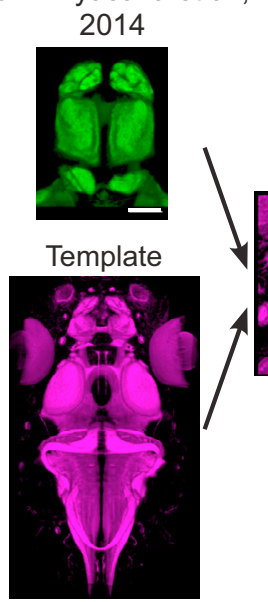

Subvolume inserted back into template
Mitral cells from

Miyasaka et al., 2014

in standard brain

Figure 2. Integration of External Data

(A) 8 examples of additional reference stains that can be used to align data to our resource. A, anterior; V, ventral. Scale bar represents $100 \mu \mathrm{m}$.

(B) Examples of Gal4-line expression patterns and single-neuron tracings from different projects aligned to our common coordinate system. ( $\left.\mathrm{B}_{1}\right)$ Enhancer-trap (ET) lines expressing UAS-Kaede from Marquart et al. (2015). $\left(\mathrm{B}_{2}\right)$ BAC-trangenic lines expressing UAS-Dendra from Förster et al. (2017). ( $\left.\mathrm{B}_{3}\right)$ ET lines expressing UAS-Dendra from Scott et al. (2007). $\left(\mathrm{B}_{4}\right)$ Enhancer-trap (ET) lines expressing UAS-GFP from Asakawa et al. (2008). ( $\left.\mathrm{B}_{5}\right)$ Various expression pattern from Randlett et al. (2015). $\left(B_{6}\right)$ Tectal projection neurons from Helmbrecht et al. (2018). All scale bars represent $100 \mu \mathrm{m}$.

(C) Integration of mitral cell tracing from Miyasaka et al. (2014). Our reference brain was cropped to match the template of the original data. After successful alignment, the cropped volume was reinserted into the full-site reference brain. The various transformation steps were also applied to the mitral cell tracings. A, anterior; V, ventral. Scale bars in subvolume represent $50 \mu \mathrm{m}$; all other scale bars represent $100 \mu \mathrm{m}$. 
A

\section{Landmarks in template}

\section{Landmarks after alignment}

\section{nuclei landmarks}

fiber landmarks

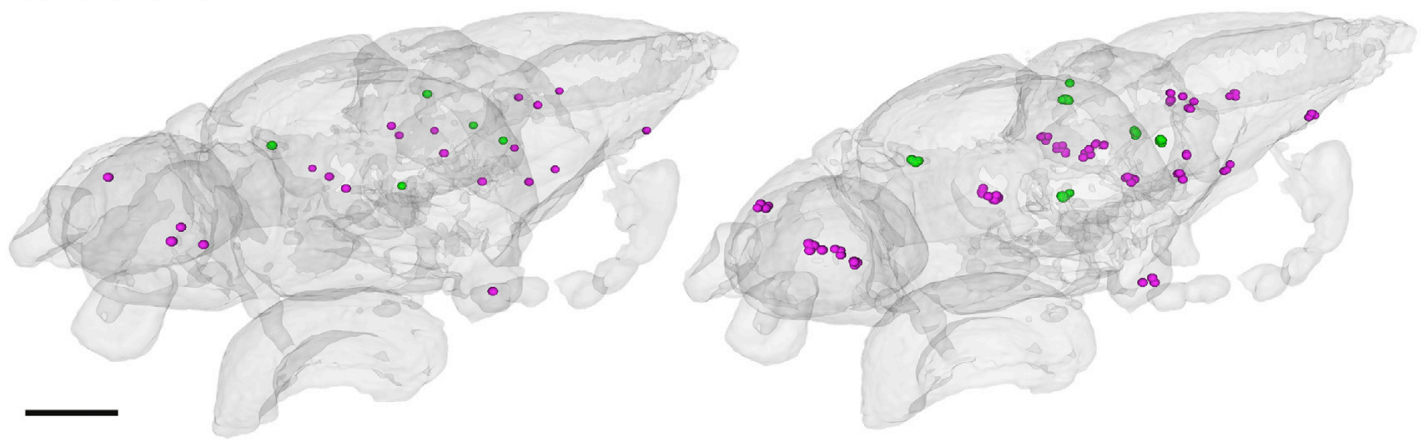

B

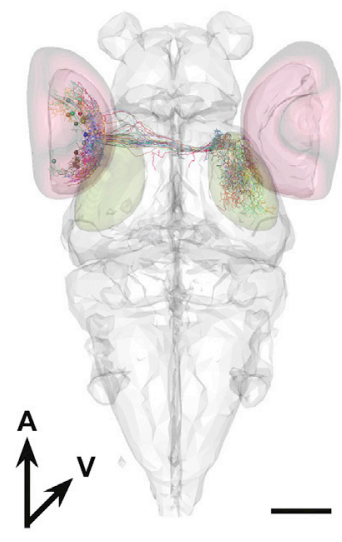

E

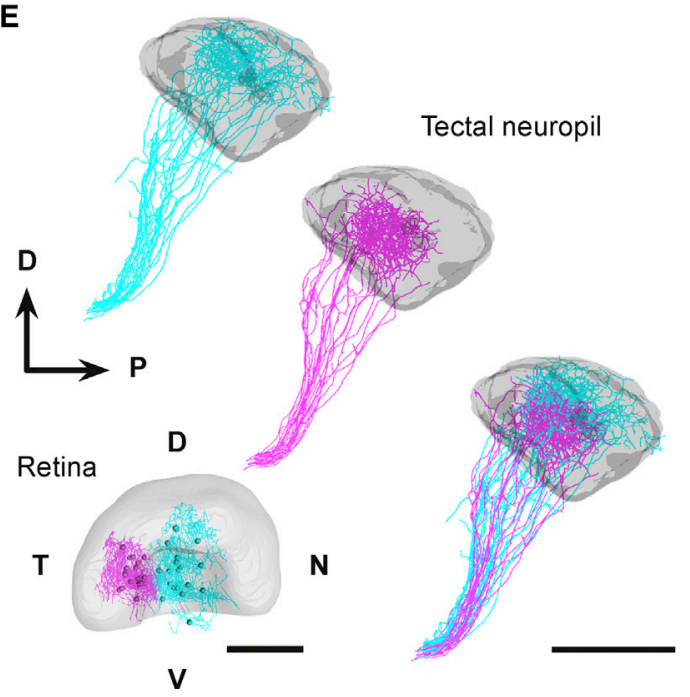

C

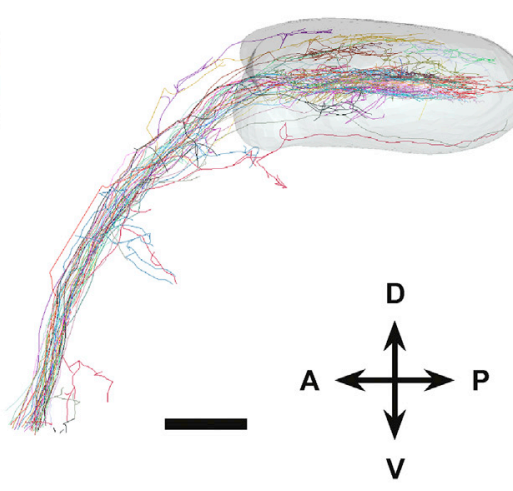

$\mathbf{F}$

SFGS 1-3

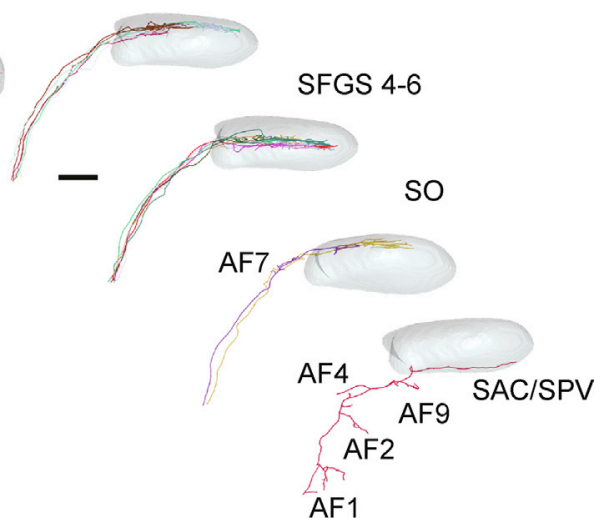

AF1

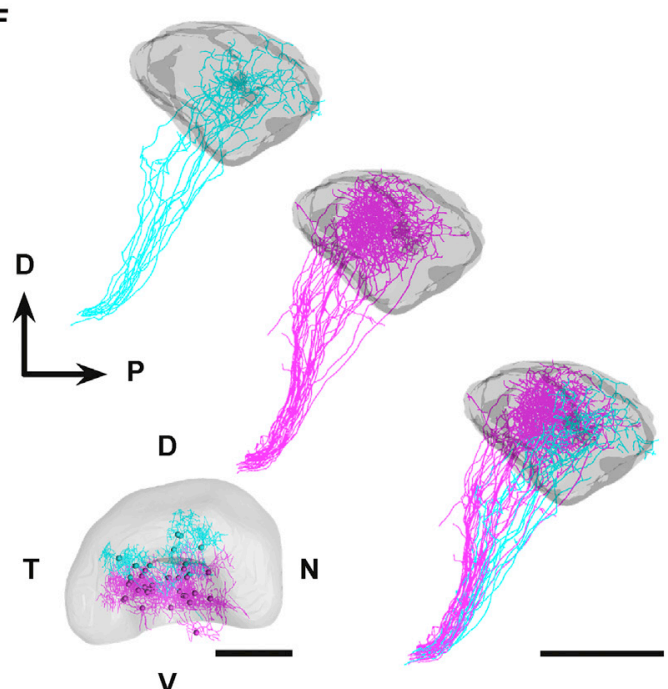

Figure 3. Verification of Registration Accuracy

(A) Left: location of fiducial points overlaid in outline of standard brain (gray). Right: location of the same fiducial points identified in original images after alignment to standard brain. Scale bar represents $100 \mu \mathrm{m}$.

(B) RGCs extracted from our database overlaid in outline of standard brain (gray). Surfaces of the retina (red) and tectal neuropil (green) are also shown. Scale bar represents $100 \mu \mathrm{m}$.

(C) Lateral view of the tectal neuropil highlighting the layered innervation of RGC. Scale bar represents $20 \mu \mathrm{m}$.

(D) Examples of RGCs identified by layer specific innervation of tectal layers and arborization fields (AFs). Scale bar represents $20 \mu \mathrm{m}$.

(E) Overlay of temporal (magenta) and nasal (cyan) RGCs in the retina (bottom left) and tectal neuropil (top left to bottom right). All scale bars represent $100 \mu \mathrm{m}$.

(legend continued on next page) 
projection-neuron tracings $(n=133)$ recently generated by our lab (Helmbrecht et al., 2018; Figure 2B6). Together the success of these efforts shows that our resource is backward compatible with previous single-cell tracing efforts, provided a bridging registration to one of the available reference markers can be established.

\section{Registration Accuracy Has Subcellular Resolution across Individual Brains}

To measure the accuracy of the registration, we then identified five landmarks within the SYP pattern (fiber landmarks) and 20 from compact cell clusters in various co-registered Gal4 patterns (nuclei landmarks), which were easily recognizable across individual brains (Figure 3A; Figure S3A), and measured the Euclidean distances to their counterparts in the standard brain (see STAR Methods). The distance varied between 0.22 and $14.13 \mu \mathrm{m}$ with most data point between 2 and $7 \mu \mathrm{m}$ and a median of $4.38 \mu \mathrm{m}$ (Figures S3B and S3C), comparable to what has been described in Drosophila (Jefferis et al., 2007). It is unknown to what extent this positional variability is biological in nature or caused by registration errors. In any case, this value corresponds to a fraction of the typical cell body diameter in larval brains $(3-7 \mu \mathrm{m})$ and confirms the large degree of stereotypy in brain architecture, which appears to extend to the positioning of individual nuclei and axon tracts. These values are derived from samples that have been inspected manually for successful alignment. For each neuron, we manually inspected the alignment of the underlying image stack (see STAR Methods).

As a test case for registration accuracy, we asked whether we could detect the well-known laminar organization of retinal ganglion cell (RGC) axon terminals in the neuropil of the optic tectum (Robles et al., 2014). Individual laminae are about 4-10 $\mu \mathrm{m}$ in thickness. When the $42 \mathrm{RGC}$ traces from our database were displayed together, individual retinotectal laminae could be discerned (Figures 3B-3D). The vast majority of RGCs in our dataset terminate in the stratum fibrosum et griseum superficiale (SFGS) layers of the tectal neuropil (36/42), most of them exclusively so (26/42). Fewer RGCs project to other layers of the tectum (4/42) and a small subset project to visual areas outside of the tectum (2/42). This distribution is similar to what has been reported in a sample of 450 RGCs traced in a previous study (Robles et al., 2014). We were also able to detect some of the more infrequent RGC types including those that have collaterals in both arborization field (AF) 7 and the tectal SO layer (Semmelhack et al., 2014), as well as a multi-collateral RGC type that projects to the tectal stratum album centrale (SAC)/stratum periventriculare (SPV) layer and multiple AFs in the thalamus and the pretectum (Robles et al., 2014).

To test whether our registration procedure retained the wellknown retinotopy of RGC terminations in the tectum, we subdivided our RGC dataset into those that originated in the nasal, temporal, dorsal, and ventral quadrants of the retina and inspected their projection sites in the tectum. We found, reassuringly, that their axons primarily terminate in the posterior, anterior, ventral, and dorsal quadrants of the tectal neuropil, respectively (Figures $3 \mathrm{E}$ and $3 \mathrm{~F}$ ), in agreement with earlier studies (Baier et al., 1996; Robles et al., 2014; Springer and Mednick, 1985; Stuermer, 1988). In conclusion, digital co-registration of 42 separately sampled RGCs in our standard brain faithfully reveals the architecture of the retinotectal projection along its retinotopic and laminar axes.

An Interactive Web Interface Enables Browsing and Searching for Neurons in Predefined Subvolumes

To make the atlas easily accessible to the community, we generated a repository (https://fishatlas.neuro.mpg.de/zebrafishatlas/ main_page\#) and programmed a user interface with the following major functionalities:

1) Visualization: neurons are initially visualized in a $3 D$ interactive browser (in this case, we choose RGCs as an example set of neurons) (Figure S4A; Video S2). The user has multiple options to change the appearance of the neurons (e.g., color, soma size, and neurite thickness) as well as the option to mirror neurons to either hemisphere of the brain for display purposes (Figure S4B1). For each neuron, a link to the metadata is provided, e.g., lab of origin, Gal4driver, cell type (if available), as well as original publication in which the neuron was described (Figure S4C1). In addition, it is possible to display the brain regions innervated by a particular neuron, with the option to limit the search to only specific parts of the neuron (Figure S4C2). Once the user has found an interesting neuron, morphologically similar neurons can be identified using NBLAST (Costa et al., 2016) (Figure S4C3). The current 3D view can be exported as an image by taking a screen shot (Figure S4D) or alternatively by downloading the tracings and using external visualization software (Figure S4B2).

2) Location-based search: to search our database for neurons in specific brain regions, we generated a basic segmentation of the larval zebrafish brain that consists of 79 brain regions covering $81 \%$ of the entire brain volume. In our example case, we selected the retina and the tectal neuropil to search for RGCs (Figure S4E; Video S3). Once the user selected one or more brain regions it is possible to perform searches on the individual regions, i.e., looking for projection neurons by searching for neurons that have their somata in the region but also terminate outside of it (Figure S4F). For more complex search function involving two or more brain regions, an advanced search function is available (Figure S4G). The user can perform logical AND/OR searches and can specify which part of the neurons is in which brain region. In our example, we searched for neurons that have their somata in the retina and fiber terminals in the tectal neuropil to find all RGCs in our dataset.

(F) Overlay of ventral (magenta) and dorsal (cyan) RGCs in the retina (bottom left) and tectal neuropil (top left to bottom right). All scale bars represent $100 \mu \mathrm{m}$. $\mathrm{A}$, anterior; AF1, arborization field 1; AF2, arborization field 2; AF4, arborization field 4; AF7, arborization field 7; AF9, arborization field 9; D, dorsal; P, posterior; SAC/SPV, boundary between the stratum album centrale and the stratum periventriculare; SFGS 1-3, stratum fibrosum et griseum superficiale 1-3; SFGS 4-6, stratum fibrosum et griseum superficiale 4-6; SO, stratum opticum; V, ventral. 
A

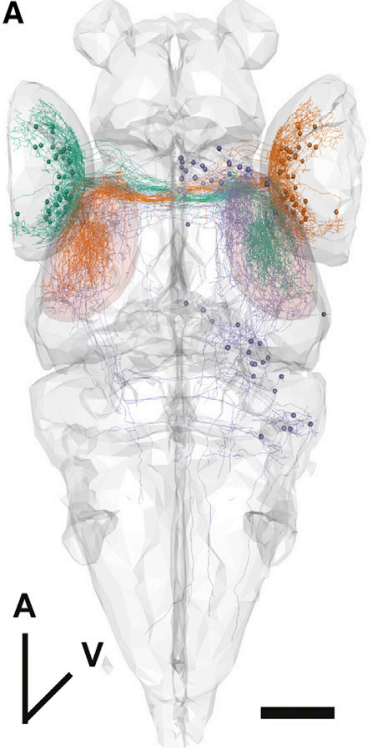

D
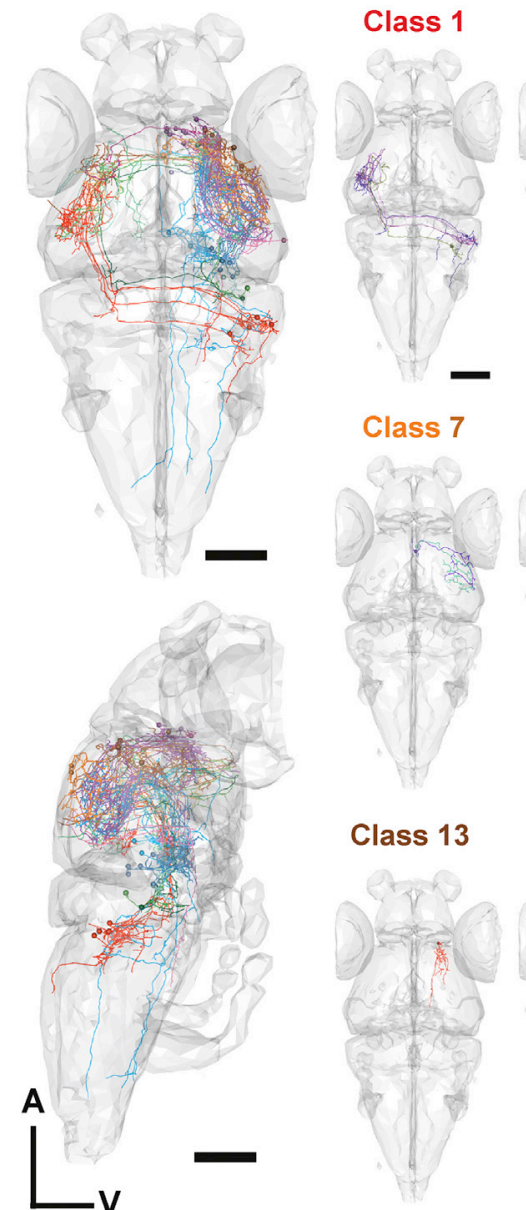

B

C

E
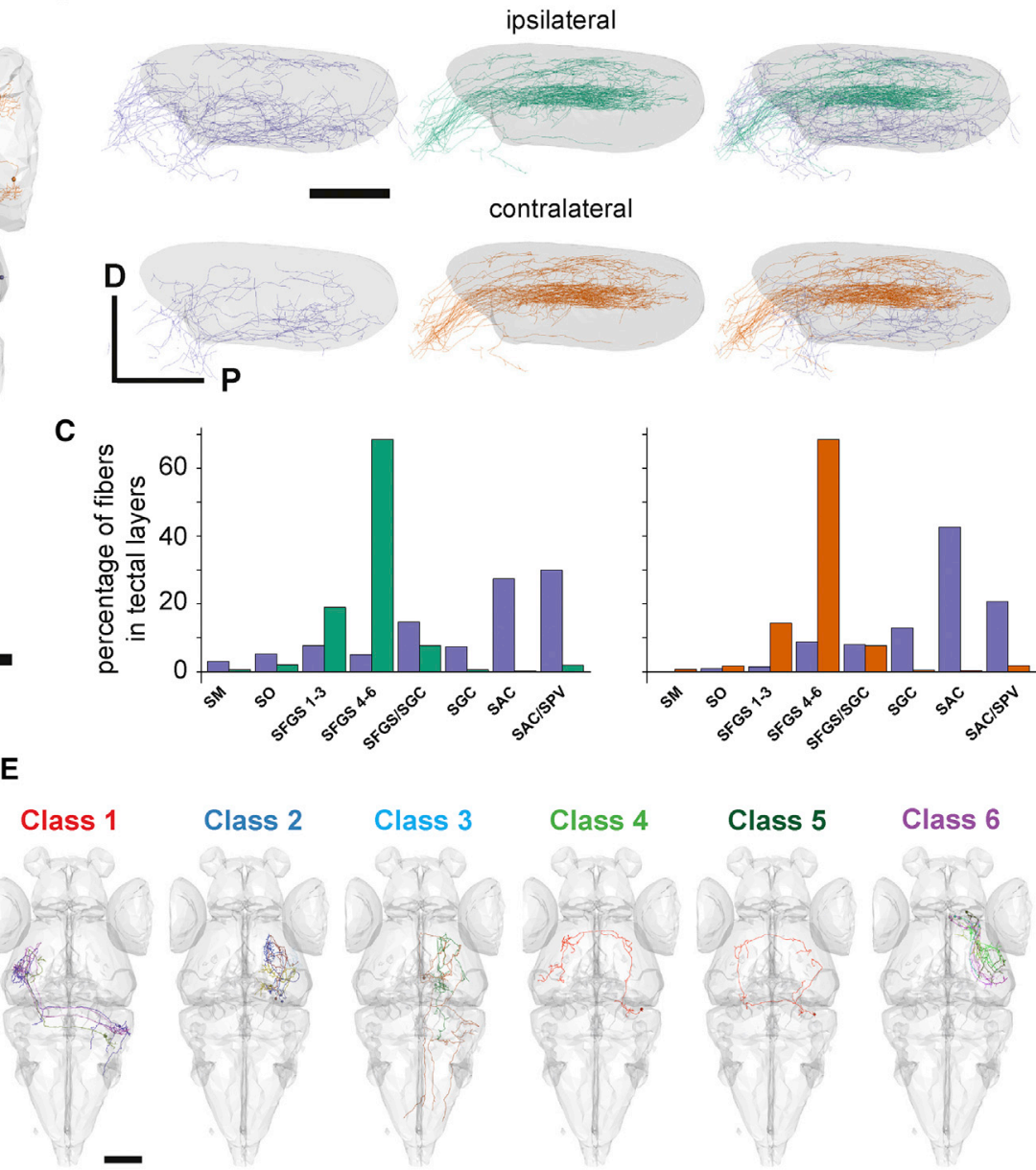

Class 5

Class 6
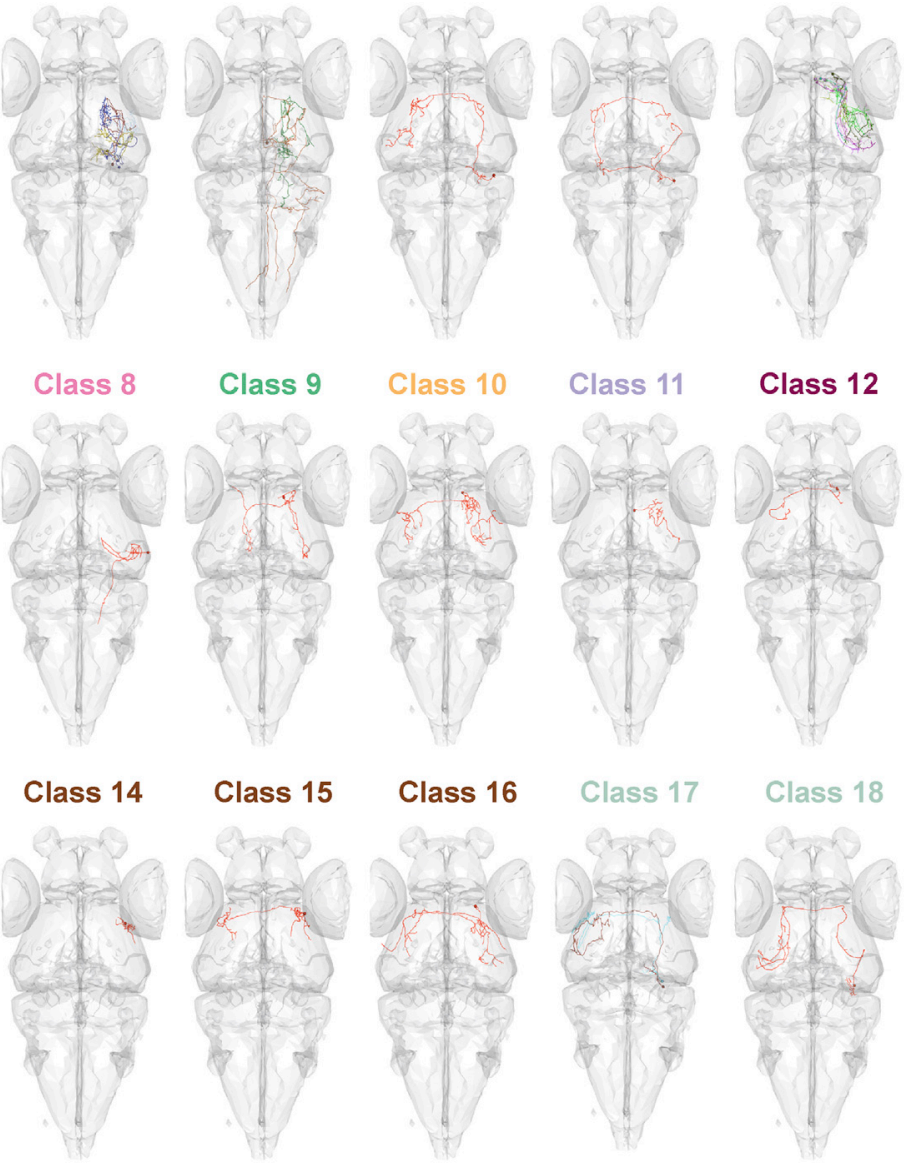

(legend on next page) 
3) Expression pattern-based search: currently, there is no consensus annotation of all the different brain regions in the larval zebrafish. One reason for that is the difficulty of defining regional boundaries due to the lack of clear anatomical demarcations. Brain regions or parts thereof can often be labeled by gene expression pattern. In order to guide the selection of specific brain regions, we aligned the unique expression patterns of $221 \mathrm{Gal} 4$ lines and other markers to our standard brain (see above). This collection currently encompasses the Gal4 patterns from the Zebrafish brain browser database (ZBB; https://science.nichd. nih.gov/confluence/display/burgess/Brain+Browser), as well as 96 new lines. In our specific example, we used a recently created bacterial artificial chromosome (BAC) transgenic line that expresses GFP in cholinergic neurons (Förster et al., 2017). Based on the GFP expression, we selectively marked the oculomotor nucleus and searched for neurons in this region (Figure $\mathrm{S} 4 \mathrm{H}$; Video S4). Often, brain regions are not defined by the expression of one marker but by the combination of multiple. To accommodate this feature, we added the possibility to visualize multiple channels simultaneously (Figure S4I).

\section{"Virtual Tract Tracing" Reveals the Spatial Organization of Multimodal Inputs to the Tectum}

We next asked whether we could employ the brain atlas to discover novel wiring principles in the tectum. To this end, we focused on the proposed segregation of visual and non-visual innervations (Basso and May, 2017; Filosa et al., 2016; Li et al., 2018; Meek, 1983; Pérez-Pérez et al., 2003). Prior work in adult teleosts had demonstrated that the non-retinal inputs tend to target different layers in the tectal neuropil than RGCs (Meek, 1983), but such laminar separation has not been investigated in the much smaller brains of zebrafish larvae. We visualized the well-described retinorecipient layers (Robles et al., 2013; Roeser and Baier, 2003; Xiao and Baier, 2007; Xiao et al., 2005) side by side with those innervated by other afferents from our dataset (Figures $4 \mathrm{~A}$ and $4 \mathrm{~B}$ ). We visualized RGCs that target the tectal neuropil (40/42) and mirrored them to each hemisphere for comparison of ipsi- and contralateral tectal innervation (green and orange, Figures $4 \mathrm{~A}$ and $4 \mathrm{~B}$ ). Non-retinal cells $(n=36)$ projecting to the tectum with soma in the left hemisphere were mirrored to the right hemisphere (purple, Figures $4 \mathrm{~A}$ and $4 \mathrm{~B})$. The majority of these afferents (22/36) originated from cells on the same side of the brain, but we also detected neurons that innervated both $(9 / 36)$ or the contralateral $(5 / 36)$ tectal hemisphere. Overlaying retinal and non-retinal axons revealed that both populations are highly segregated within the tectal neuropil: while RGC axons are innervating the superficial layers, mainly SFGS, as reported before, non-retinal inputs primarily target deeper layers, such as SAC, in which RGC axons are absent (Figure 4C).

We analyzed this dataset at the individual neuron level to determine the origin of non-retinal input. We manually subdivided the 36 non-retinal afferents in 18 classes, based on soma location and projection pattern (Figure 5D) and generated a wiring diagram of non-retinal tectal input (Figure S5). Most classes contain only one neuron, but others have up to 9 (class 6; Figure 4D). The majority of cells originate in the thalamus (10/37), and a recent manuscript described these as sensitive to looming and luminance decrease (Heap et al., 2018). In addition to the thalamus, other prominent input arises from the nucleus isthmi $(\mathrm{NI}, 8 / 37$, class 2,17 , and 18) as well as the medial octavolateralis nucleus (MON, 4/37, class 1). The latter area receives inputs from lateral line neuromasts and is sensitive to water currents (Thompson et al., 2016). In total, we identified 15 distinct regions that innervate the tectum (Figure S5), including some that process sensory information (e.g., MON and thalamus) or the internal state (e.g., intermediate hypothalamus and reticular formation). Taken together, anatomical data extracted from our brain atlas corroborate a function of the tectum in multimodal integration of sensory information and internal state.

\section{The Brain Atlas Reveals a Topographic Organization of Cerebellar Projections}

The cerebellum of vertebrates is involved in coordinated smooth movements and motor learning. One of the organizing principles proposed for the cerebellum is functional regionalization (Manni and Petrosini, 2004). In zebrafish, two similar visual stimuli that trigger distinct motor outputs (optokinetic and optomotor responses) have been shown to evoke region-specific activity of Purkinje cells (Matsui et al., 2014). In addition, a topographical map for processing of different sensory modalities was found in granule cells (Knogler et al., 2017). To find evidence for a topographic organization of the output pathways, we investigated the neurons that projected out of the cerebellum. There are two cell types that provide output for the cerebellum, Purkinje cells (PCs) and eurydendroid cells (ECs). PCs exclusively send their projection to the MON, while ECs provide a more diverse output of the cerebellum (Bae et al., 2009).

Figure 4. Analysis of Non-retinal Tectal Afferents

(A) Overlay of all RGCs mirrored to the right (orange) and left (green) hemisphere and all non-retinal afferents (purple) mirrored to the right hemisphere in outline of standard brain (gray). Scale bar represents $100 \mu \mathrm{m}$.

(B) Lateral view of the tectal neuropil highlighting the anatomical segregation of retinal and non-retinal input to the ipsi- and contralateral tectum. Scale bar represents $50 \mu \mathrm{m}$.

(C) Bar plots displaying the percentage of overall fiber length in the different layers of the ipsi- and contralateral tectum for retinal (green and orange) and nonretinal afferents (purple).

(D) Overlay of all non-retinal afferents mirrored to the right hemisphere in outline of standard brain (gray). Different colors represent the different classes. All scale bars represent $100 \mu \mathrm{m}$.

(E) Individual classes of non-retinal afferents displayed side by side in outline of standard brain (gray). Scale bar represents $100 \mu \mathrm{m}$. A, anterior; D, dorsal; P, posterior; SAC, stratum album centrale; SAC/SPV, boundary between the stratum album centrale and the stratum periventriculare; SFGS, stratum fibrosum et griseum superficiale; SFGS/SGC, boundary between the stratum fibrosum et griseum superficiale and the stratum griseum centrale; SM, stratum marginal; SO, stratum opticum; V, ventral. 


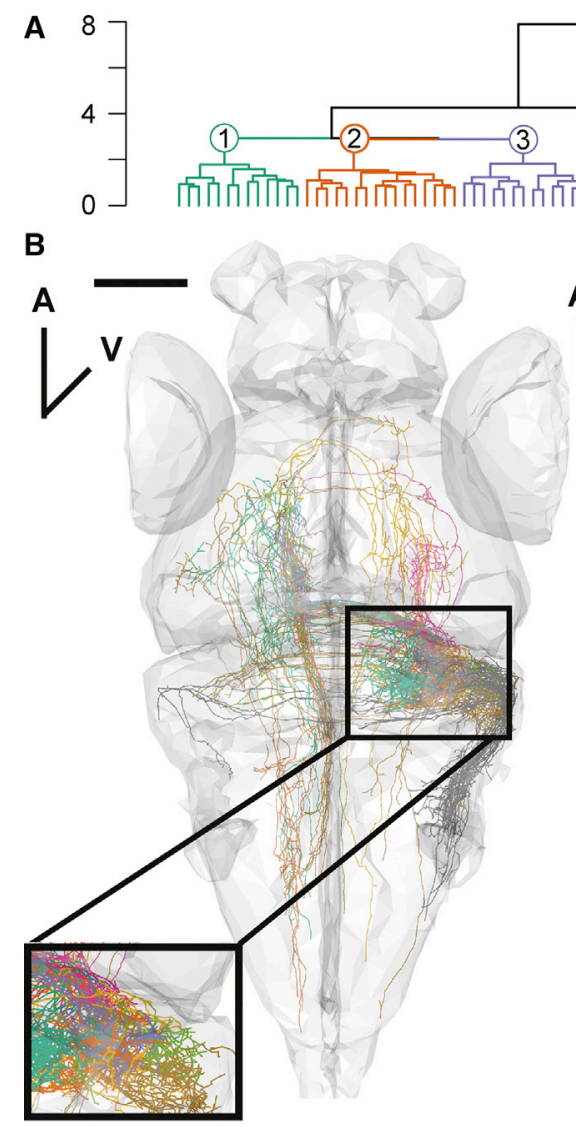

Cluster 3

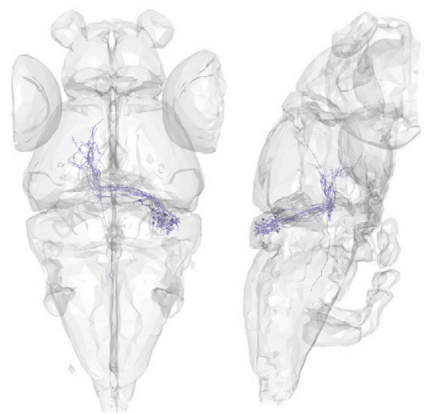

Cluster 6
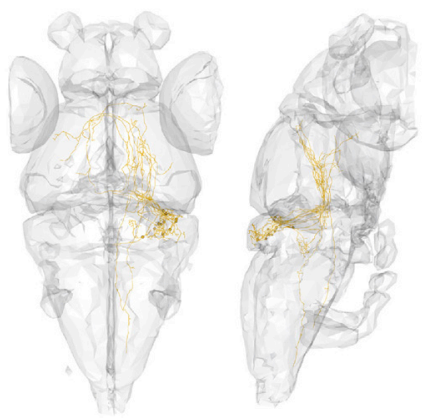

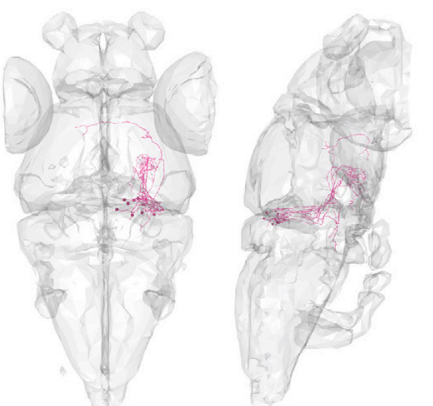

Cluster 7
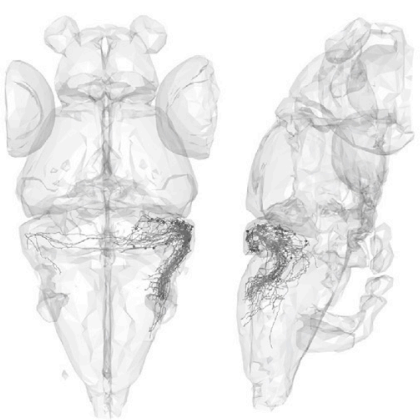

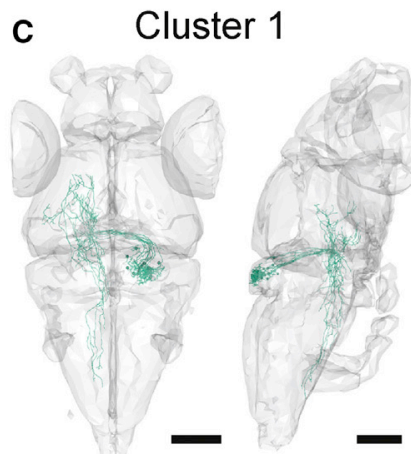

Cluster 2

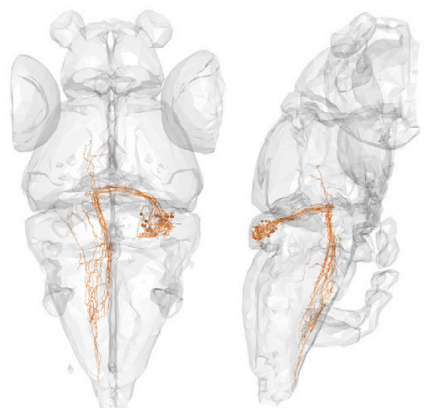

Cluster 5

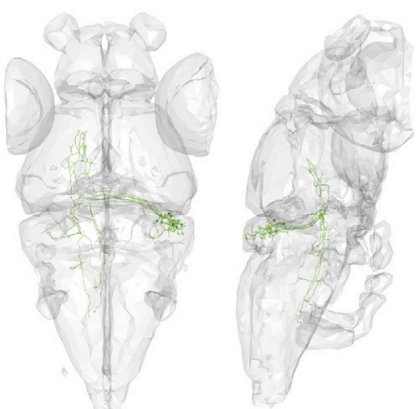

Cluster 8

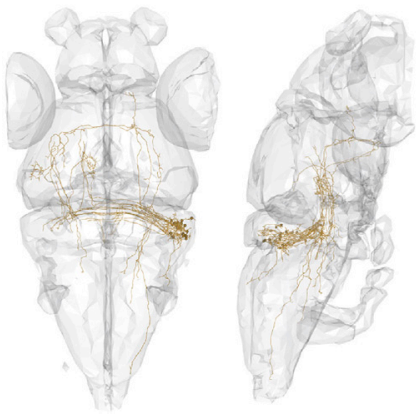


We found 121 cerebellar output cells, whose cell bodies reside along the medio-to-lateral and rostral-to-caudal extent of the cerebellar corpus. The majority of those are ECs (91/121) and the rest are PCs (30/121). Their projections extend to a variety of brain regions, including the thalamus, the hypothalamus, the tegmentum, the nucleus of medial longitudinal fascicle, the oculomotor and trochlear nucleus, as well as the reticular formation and the medulla oblongata (Figure S5A). The NBLAST algorithm (Costa et al., 2016) was used to hierarchically cluster the individual cells based on their morphological similarity (Figure 5A). Of the eight major clusters, one (cluster 7) consisted exclusively of all PCs, with the remaining ECs distributed over the other seven clusters. Color coding these clusters (Figures $5 \mathrm{~B}$ and $5 \mathrm{C}$ ) revealed that the dendrites of the individual $E C$ clusters tile the granule cell layer, suggesting segregation of inputs. Interestingly, the different clusters projected to distinct target regions (Figure S6A). Dendrites of clusters 1, 2, and 3 are arrayed topographically along the medial-to-lateral axis at the same rostro-caudal level; this order is preserved, albeit flipped, for their axon projections in the tegmentum (Figure S6B). We observed a different pattern for EC connections to the thalamus. Here, dendrites of clusters 1, 3, and 5 show almost perfect tiling in the granule layer, yet their axons intermingle within the thalamus (Figure S6C). Cluster 4 ECs are distinct from other clusters in that they provide the sole input to the ipsilateral intermediate hypothalamus (Hi), as well as the majority of inputs to the ipsilateral tegmentum. Such topographic relationships provide entry points into studies of the cerebellum's functional specializations, as they may guide experimental interventions to particular sites in the brain.

\section{Neurons Are Classifiable Based on Their Locations and Morphologies Using NBLAST}

We classified all 2,143 neurons (including mitral cells and tectal projection neurons) by morphology and innervation pattern using NBLAST. Similarity scores were calculated to perform hierarchical clustering (Figures 6A and 6B; Video S5). Looking at the dendrogram, it becomes apparent that separable clusters of neurons exist. When we cut the resulting dendrogram at a height of 8 we end up with 22 distinct clusters. At this cutoff, we can already see clusters identical to known cell classes (Figure 6C). Examples are cluster 13 (oculomotor neurons) and cluster 14 (RGCs). However, at this cutoff, many clusters are still heterogeneous. For example, cluster 12 consists of multiple neuron types located in the forebrain. When we lowered the cutoff to 3 , cluster 12 contained at least 6 distinguishable morphological types ("morphotypes"; Figure 6D). Three of those are part of the olfactory pathway. Sub-cluster 12-2 consists of olfactory receptor neurons (ORNs) that convey information from the olfactory epithelium to the olfactory bulb (OB) and sub-cluster 12-4 consists of mitral cells (see Figure $2 \mathrm{C}$ for comparison). Sub-cluster 12-1 is composed of local interneurons of the OB that modulate information transfer between the ORNs and the mitral cells. In addition, we could also find three clusters of pallial and subpallial neurons (sub-cluster 12-3, 12-5, and 12-6) that show strong overlap with the projections of the mitral cells and could potentially be downstream targets.

The clustering approach successfully isolates well-known cell types and discovers apparently new ones. Three examples of known types are neurons that provide input from the ventral entopeduncular nucleus to the habenula (Turner et al., 2016) (vENT-Hb; Figure S7A1), habenular output neurons that project to the interpeduncular nucleus (NIn) and the superior raphe (Amo et al., 2010) (Hb_PN; Figure S7A2), as well as tangential neurons that provide input from the tangential nucleus to the oculomotor nucleus and the nMLF (Bianco et al., 2012) (Figure S7A3). Among the novel types are tegmental neurons that project to the hypothalamus and pallium (T-Hi/P, Figure S7B1), neurons of the $\mathrm{NI}$ that innervate the thalamus, torus semicircularis, and the reticular formation (NI-RF/TS/Th; Figure S7B2), as well thalamic neurons that project to the hypothalamus and the medial part of the reticular formation (Th-Hr/mRF; Figure S7B3).

Lowering the threshold also leads to a splitting up of distinct cell types into further sub-clusters that appear not to reflect bona fide cell types. For instance, RGCs can be further subdivided based on their dendritic morphology and axonal projection pattern (Robles et al., 2014), and, when we lowered the height at which the dendrogram was cut, RGCs were split up in separate clusters (Figure S7C). However, the difference between individual clusters primarily reflected the position of the cell bodies and dendrites within the retina and the respective retinotopic mapping of the axons in the tectal neuropil, rather than collateral patterns or laminar stratification. In summary, our catalog of cell morphologies can be automatically organized using NBLAST leading to the classification of cell classes. However, such first-pass results have to be carefully interpreted, as NBLAST tends to weight cell-body location and neurite orientation more heavily than morphological parameters.

\section{Combing Single-Cell Tracings with Annotation of Brain Regions Generates a Mesoscale Connectome}

To generate a brain-wide undirected connectivity matrix, we divided the brain into 36 non-overlapping regions at approximately similar ontological level (Figure 7). These regions cover $\sim 82 \%$ of the brain volume. Since the regions are bilaterally symmetric, we divided them into a left hemispheric region and a right one, for a total of 72 brain regions. While this annotation is not exhaustive, we can use it to generate a mesoscale connectome, which will evolve with further knowledge of anatomical boundaries and the addition of more cells. We chose an undirected connectivity matrix since the distinction of dendritic and axonal arbors is not always as obvious as in the previous examples shown in Figures 4, 5, S5, and S6.

Figure 5. Topographic Organization of Cerebellar Output

(A) Hierarchical clustering (HC) of cerebellar efferents based on NBLAST similarity scores. Colors indicate the different clusters.

(B) Overlay of all cerebellar efferents mirrored to the right hemisphere in outline of standard brain (gray). Different colors represent the different clusters from (A). Black rectangles represent regions zoomed in. PCs (cluster 7) were omitted for clarity. Scale bars represent $100 \mu \mathrm{m}$.

(C) Individual classes of cerebellar efferents displayed side by side in outline of standard brain (gray). All scale bars represent $100 \mu \mathrm{m}$. A, anterior; $\mathrm{V}$, ventral. 
A

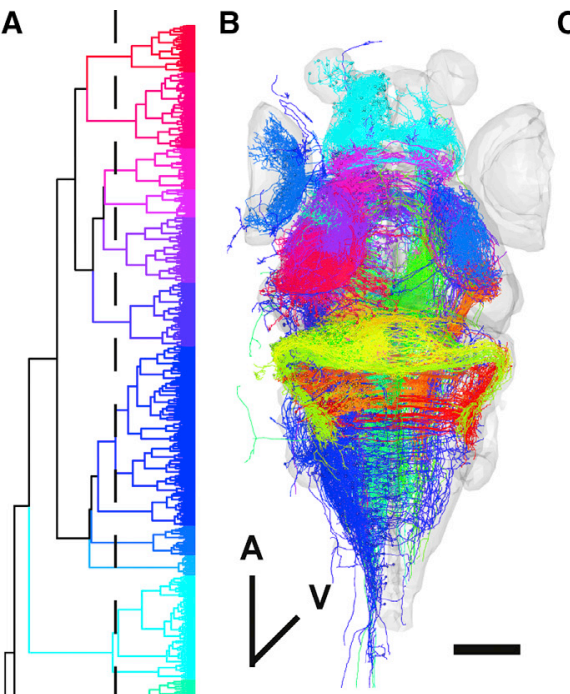

Cluster 11 Cluster 12 Cluster $13^{*}$

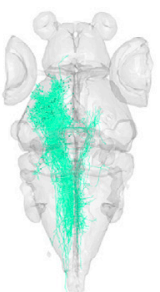

Cluster 18
C Cluster 1

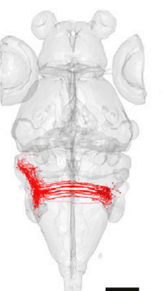

Cluster 6
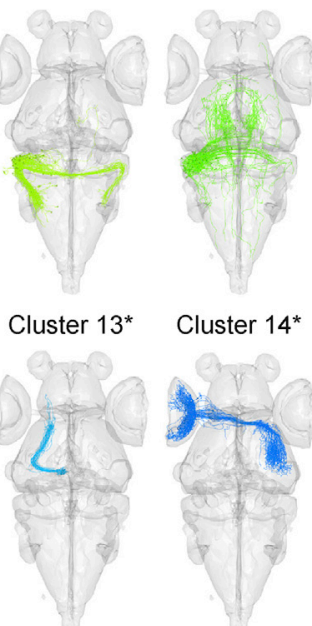

Cluster 20

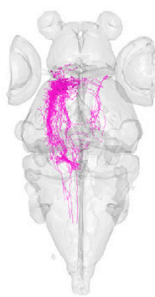

Cluster $14^{*}$

Cluster 2

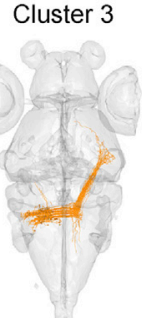

Cluster 8

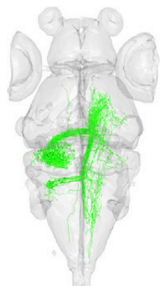

Cluster 15

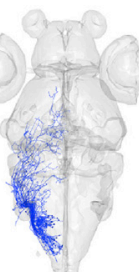

Cluster 22
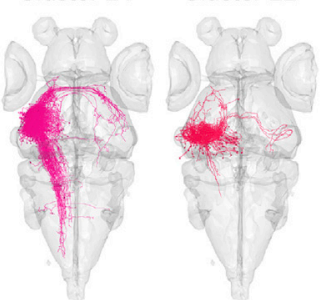

Cluster 4

Cluster 5

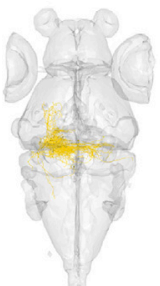

Cluster 9 Cluster 10
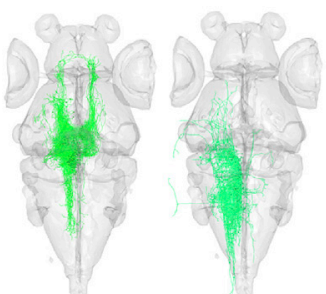

Cluster $16 \quad$ Cluster 17
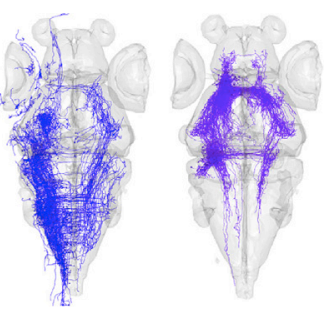

D
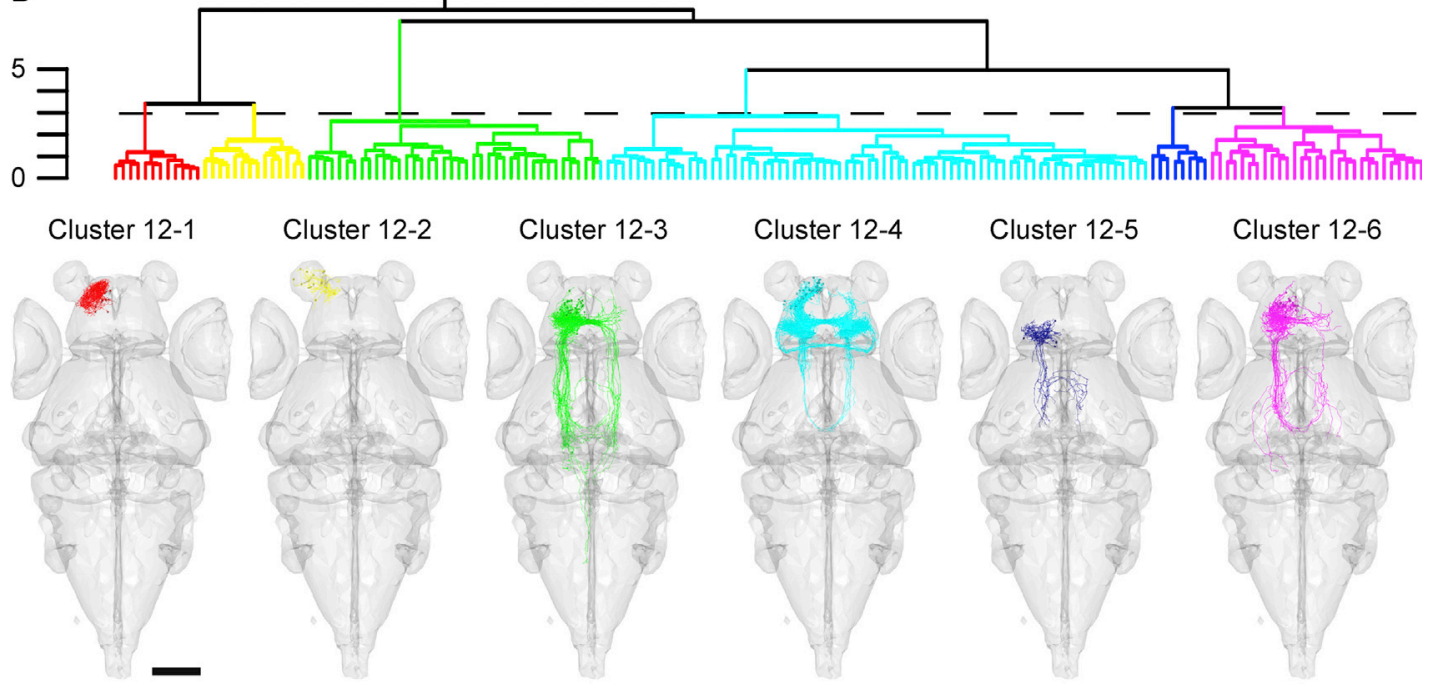
Connection strengths between individual brain regions were calculated from the total sum of neurite lengths in each of the possible combination of two brain regions from all neurons that connect them (see STAR Methods). To prevent biases in connection strength arising from different sizes, we normalized connection strength by the total volume of the two brain region pairs. This approach revealed connection strengths that span a range greater than $10^{5}$-fold across the brain. The observed connection strength follows a lognormal distribution (Figure 8A) and decays exponentially as a function of distance (Figure 8B; $R=-0.39$ ) in accordance with previous results from other animals (Betzel and Bassett, 2018; Ercsey-Ravasz et al., 2013; Oh et al., 2014) and consonant with an economic network organization, which minimizes wiring lengths (Bullmore and Sporns, 2012).

The resulting connectivity matrix allowed us to perform hierarchical clustering of brain regions by similarity of input and outputs (Figure $8 \mathrm{C}$ ). For each brain region, the 3D center of masses was plotted in our standard brain (Figure 8D; Video S6), with the colors reflecting the clusters of Figure $8 \mathrm{C}$. We also plotted the connectivity matrix as a $3 \mathrm{D}$ wiring diagram, with the size of the nodes representing the node degree (number of neurons that connect to or from that brain region) and the thickness of the connections representing the normalized connection strength (Figure 8E; Video S7). Various levels of organization become immediately apparent indicating a non-random organization. Closer inspection reveals two main clusters. One (cluster 1 , red) consists of nodes that show a high degree of connectivity that strongly participate in the overall network structure. This cluster consists mainly of the subdivisions of the reticular formation and the raphe nucleus. The other clusters (white rectangle) are more heterogeneous. For example, cluster 2 (green) consists mostly of peripherally located ganglia that convey sensory information to specific central brain regions or motor information to the muscles but has fewer and weaker connections to the rest of the network (e.g., olfactory epithelium or vagal motor neurons). In addition, we also find strongly reciprocally connected brain regions. For example, the two main regions of the telencephalon (pallium and subpallium) form strong connections with each other and their respective counterpart on the contralateral hemisphere as well as the preoptic region of the hypothalamus and the posterior tuberculum (cluster 3 , cyan).

\section{DISCUSSION}

The larval zebrafish is increasingly being used to address fundamental questions of nervous-system organization and circuit function at the whole-brain level (Vanwalleghem et al., 2018), yet its neuro-anatomy is still relatively uncharted. Here, we introduce a novel resource, which will be beneficial to approaches that attempt to tie neuronal function to the structure of the under- lying circuitry. We generated a dataset of 1,955 single-neuron tracings, which we aligned to an average standard brain. We verified, using a set of stereotyped anatomical landmarks, that registration accuracy is at subcellular resolution $(\sim 4 \mu \mathrm{m})$ and faithfully recapitulates known wiring principles such as retinotopic organization and layer-specific innervation of RGC axonal arborizations. To ensure cross-platform compatibility and to enable submission of external data into our platform, we registered additional reference stains to the standard brain, including commonly used antibody stains and calcium indicator transgene expression patterns. Single-cell data from subvolume imaging experiments can be incorporated with little effort, as demonstrated by the successful addition of $55 \mathrm{OB}$ mitral cells previously reconstructed by a different lab (Miyasaka et al., 2014), as well as additional 133 single-neuron tracing previously published (Helmbrecht et al., 2018). We enriched our dataset with a collection of Gal4 and GFP transgenic expression patterns (Förster et al., 2017; Marquart et al., 2015; Randlett et al., 2015; Scott et al., 2007). Earlier work used EM tracings of neurons with myelinated axons (Hildebrand et al., 2017). This dataset was largely limited to peripheral and spinal projection neurons and therefore complements this resource.

Our constantly growing dataset can be viewed online (https:// fishatlas.neuro.mpg.de/zebrafishatlas/main_page\#). This web portal allows to interactively search for neurons and visualize them in relation to other neurons, commonly used marker stains, and transgene expression patterns. The complete toolbox of searching, browsing, and viewing features is available to the community and will be expanded to accommodate other data modalities. For instance, the here-presented resource will be useful for researchers who wish to search for anatomical connections between functionally correlated regions in whole-brain imaging studies (Ahrens et al., 2013; Portugues et al., 2014). Our database has been used successfully by an accompanying study (Kramer et al., 2019) to complement GCaMP6 imaging studies investigating neuronal circuits processing optic flow. The authors could find long-range projection neurons that connect the pretectal area to the cerebellum and the reticular formation, providing a sensorimotor pathway suggested to drive behavioral responses to optic flow.

To facilitate data mining and discovery of novel neuronal cell types, we analyzed our dataset using the NBLAST algorithm, which was developed for a large single-neuron dataset of fruit flies (Costa et al., 2016). An all-by-all comparison of the entire dataset provided us with a morphological similarity matrix on which we performed hierarchical clustering. By adjusting the height at which to cut the resulting dendrogram, we were able to identify multiple already known cell classes, confirming the robustness of NBLAST. We also discovered several novel types by inspection. However, such first-pass results have to be carefully interpreted, as NBLAST tends to weight cell-body and neurite

Figure 6. Organization and Classification of Neurons Using NBLAST

(A) HC of all neurons based on NBLAST similarity scores. Colors indicate the different clusters. Dashed line indicates the height at which the dendrogram was cut. (B) Overlay of all neurons mirrored to the right hemisphere in outline of the standard brain (gray). Different colors represent the different clusters from (A).

(C) Individual clusters of neurons displayed side by side in outline of the standard brain (gray). Asterisks denote cluster of known cell types.

(D) $\mathrm{HC}$ of neurons from cluster 12 in (C). Colors indicate the different clusters. Dashed line indicates the height at which the dendrogram was cut. All scale bars represent $100 \mu \mathrm{m}$. A, anterior; V, ventral 


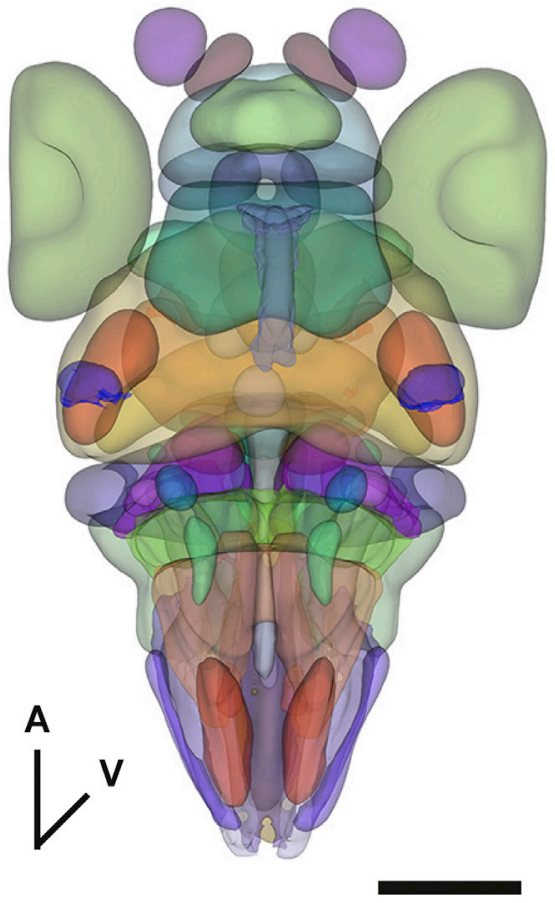

T

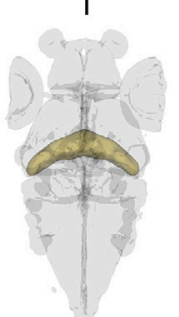

MOS1

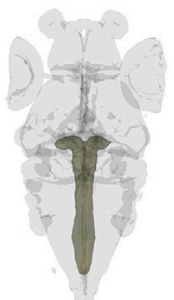

NX

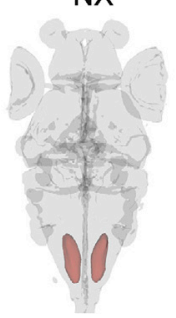

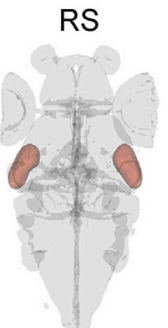
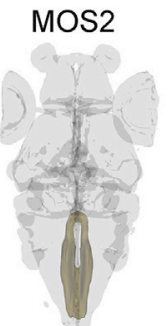

NIn

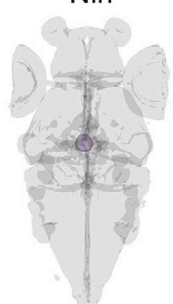

MOS3

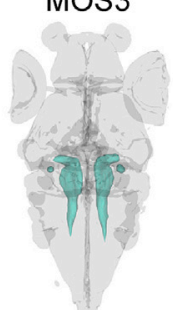

GG
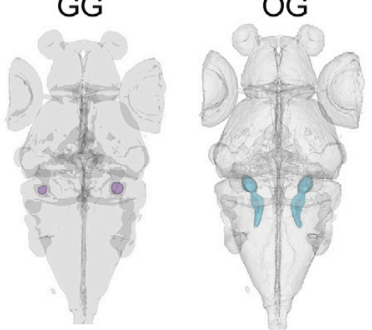

PT

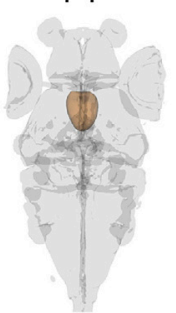

Th

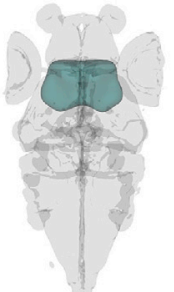

$\mathrm{Hr}$

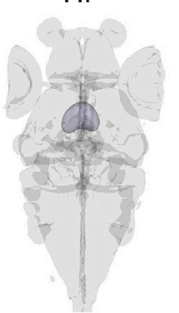

Pr

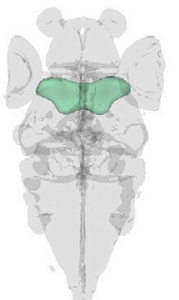

$\mathrm{Ce}$

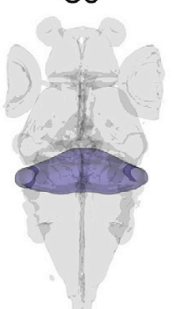

MOS4

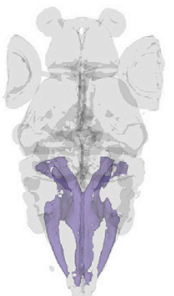

Ret

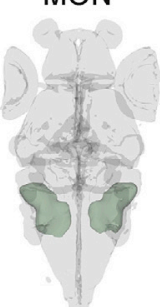

MOS5

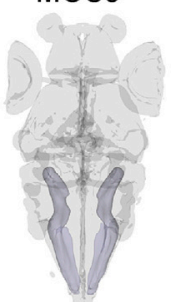

TG

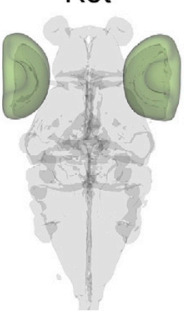

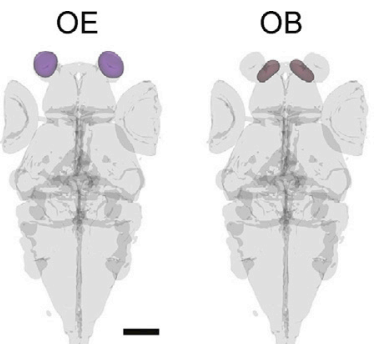

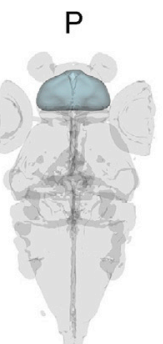

$\mathrm{Hi}$

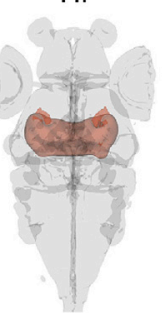

$\mathrm{Ha}$

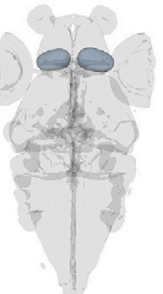

aRF

S

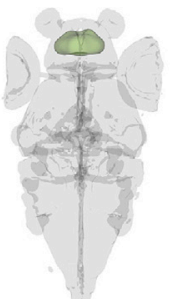

$\mathrm{Hc}$

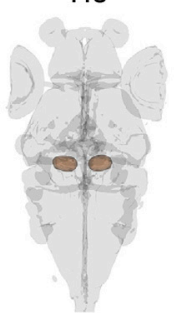

$\mathrm{TeO}$

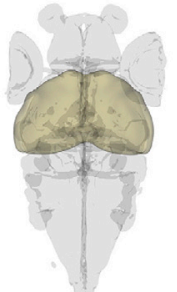

iRF

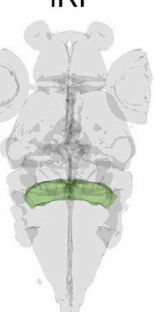

R

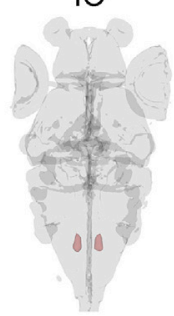

Po

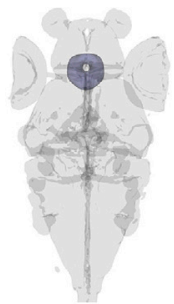

Pit

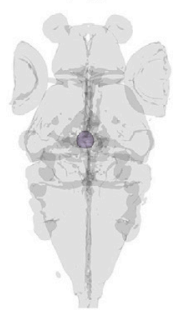

TL

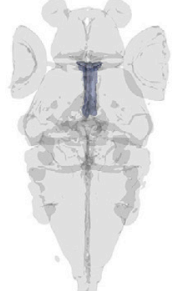

pRF

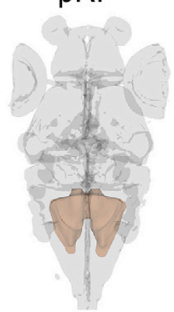

VR

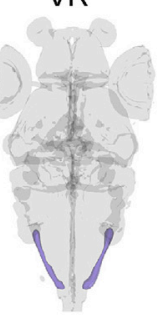

Figure 7. Brain Annotation for Graph Theoretical Analysis

36 brain regions were manually defined. Different colors for the brain regions are selected randomly. For explanation of brain region abbreviations, see Table S1. All scale bars represent $100 \mu \mathrm{m}$. 
A

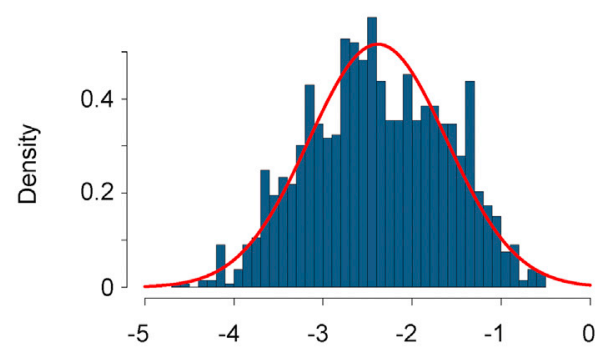

B Normalized connection strength $\left(\log _{10}\right)$

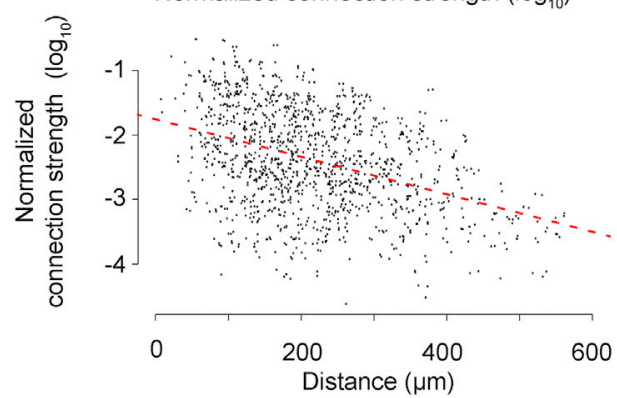

c
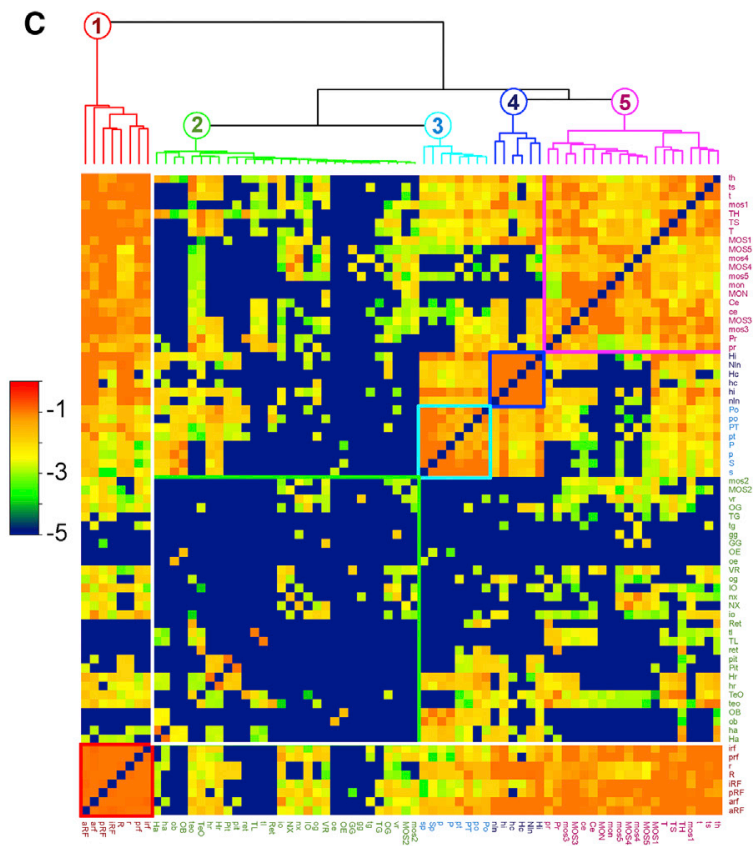

E

D
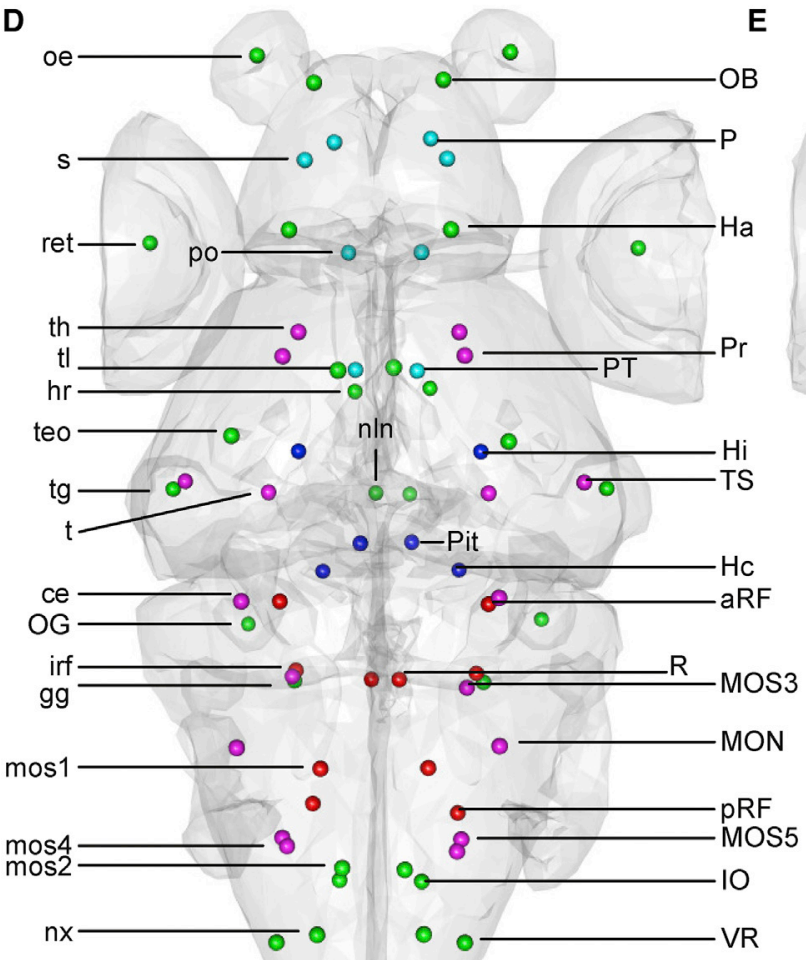

$\overbrace{}^{A}$

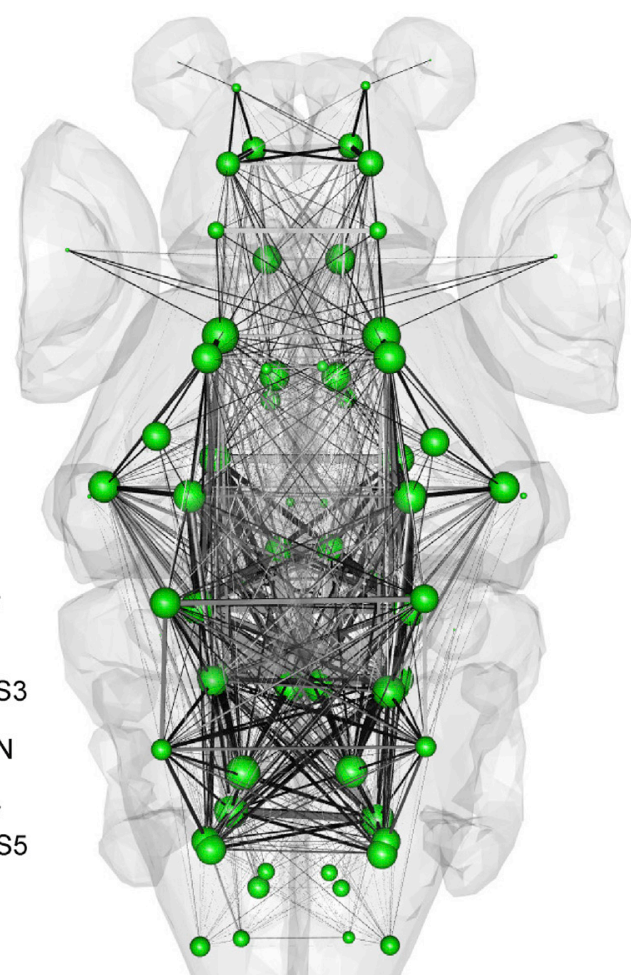

(legend on next page) 
location more strongly than intrinsic morphological parameters. Moving forward, we envision this database to become an openaccess repository similar to http://neuromorpho.org/ (Akram et al., 2018; Ascoli et al., 2007). While a comprehensive celltype census is more likely to come from genetic expression profiling, such single-cell RNA sequencing (McKenna et al., 2016; Pandey et al., 2018; Raj et al., 2018), a morphological classification of zebrafish neurons, ideally with their transcriptional profiles and developmental lineages, will be tremendously useful for experimental and computational neuroscientists alike.

To gain insight into the general architecture of the larval zebrafish brain, we constructed a cellular-resolution mesoscale connectome. For this, we divided the brain into 72 non-overlapping brain regions and determined their pairwise connection strengths to generate an undirected graph that we subjected to further analysis. Already at the current stage of our database with only about $2 \%$ of the entire neuronal complement covered, several organizational principles become evident. The interareal connection strengths exhibit a lognormal distribution and are decaying exponentially with distance. This is consistent with the theory of economic brain network organization, which states that brains are dominated by short-range connections to minimize wiring costs (Bullmore and Sporns, 2012). We found that the larval zebrafish brain displays a core-periphery organization with brain regions mainly related to sensory or motor function falling into one module and more central regions involved in higher-order integration falling into other modules. For example, we found that the forebrain together with the preoptic region and the posterior tuberculum comprises a distinct module anatomically separated from the mid- and hindbrain, which seem to be much more strongly interconnected. This agrees with a wholebrain imaging experiment that reported stronger correlation of spontaneous activity between mid- and hindbrain compared to the forebrain (Ahrens et al., 2013). We do caution in overinterpreting our results since a large number of morphotypes still need to be described. However, given the simplicity of our workflow and the ease with which data from other labs can be incorporated into our standard brain (as illustrated by the mitral cells), a complete census will be accomplished in the next few years. In addition, with a growing dataset it may become possible to apply machine learning algorithms to differentiate between axons and dendrites (Lee et al., 2014).

To demonstrate the immediate utility of our dataset, we performed virtual tract tracing of the afferent fibers innervating the tectum. First, we investigated the laminar organization of modality-specific inputs and showed that RGC and non-retinal axons are segregated, with RGCs targeting medial to superficial layers and non-retinal fibers primarily targeting deeper layers. This is in accordance with the situation in adult goldfish (Meek, 1983). When we further investigated the individual cells that provide non-retinal inputs, we identified 18 different cell classes. The most prominent non-retinal input to the tectum arises from the thalamus. Such thalamo-tectal neurons have recently been reported to respond to dimming of the visual field (Heap et al., 2018). Another prominent input arises from the contralateral MON, which receives sensory input from the lateral line and the inner ear (Liao and Haehnel, 2012; McCormick, 1989). Consistent with this anatomical arrangement, functional imaging detected responses to contralaterally applied water flow and auditory stimuli in the deeper layers of the tectum (Thompson et al., 2016). We further showed that the majority of these axons also branched in the torus semicircularis (Fame et al., 2006), a feature that was not known from previous work. The tectum is from a network perspective ideally located to connect sensory areas directly with motor areas. Yet it is also well enough integrated with the rest of the brain so that information from other brain regions can exert influence over sensorimotor transformations taking place in the tectum. This principle of extrinsically modulated "labeled lines" was demonstrated for the decision to approach or avoid a visual object based on its size. Larval zebrafish chase after small, prey-like dots and escape from large ones (Barker and Baier, 2015). The tectal output channels that steer the animal's movements toward or away from the object are anatomically segregated (Helmbrecht et al., 2018). The decision between approach and avoidance is mediated by the tectum, and hunger stimuli from the serotonergic system can shift the animal's behavioral tendency to approach larger stimuli that would normally evoke escapes (Filosa et al., 2016). Our dataset revealed additional inputs from central brain regions (PO, $\mathrm{PT}, \mathrm{T})$, which may modulate this decision-making process.

Our dataset allowed us to describe, for the first time, the topographic organization of the cerebellar projection neurons. Teleosts lack the deep cerebellar nuclei of mammals; instead, they have a specialized cell class, the ECs, that receive direct input from PCs and project widely to other brain regions (Bae et al., 2009). The collection of $91 \mathrm{ECs}$ from our database innervate a large proportion of the mid- and hindbrain. Using NBLAST (Costa et al., 2016), we divided the ECs into seven different classes. Closer inspection of their projection patterns revealed a topographical organization. Their dendrites tile anatomically compact regions of the granule cell layer, and their axons project to distinct, yet partially overlapping, brain regions. This topographical output provides an anatomical substrate for how

Figure 8. A Brain-wide Wiring Diagram of the Larval Zebrafish Brain

(A) Distribution of normalized connection strengths between brain regions.

(B) Correlation between internodal distance and normalized connection strength $(R=-0.39, p<2.2 e-16)$.

(C) Inter-region connectivity matrix of the larval zebrafish brain, derived from normalized connection strengths between brain regions. Brain regions in lowercase are located in the left hemisphere and brain regions in uppercase on the right. Brain regions with a similar connection profile are clustered together. For explanation of brain region abbreviations, see Table S1.

(D) Brain regions displayed as center of masses in outline of standard brain (gray). Colors represent cluster from (C). For explanation of brain region abbreviations, see Table S1.

(E) Brain-wide wiring diagram of the larval zebrafish brain. Location of brain regions is the same as in (D). Size of nodes reflects the node degree and thickness of edges the normalized connection strengths. All scale bars represent $100 \mu \mathrm{m}$. A, anterior; V, ventral. 
regionalized PC activity gets converted into distinct motor patterns as was demonstrated for the optomotor and optokinetic reflexes (Matsui et al., 2014). Taken together, the high-fidelity coregistration feature of our resource makes it possible to carry out virtual tract tracing studies to test hypotheses in silico and discover new wiring principles.

Another area of investigation that will benefit greatly from this new resource is dense electron-microscopic (EM) reconstructions of circuit structure (Denk and Horstmann, 2004; Hildebrand et al., 2017; Ohyama et al., 2015; Varshney et al., 2011; Zheng et al., 2018). The tracing of fibers over hundreds or thousands of ultrathin sections requires high spatial resolution and image contrast and is notoriously prone to continuation and merging errors, especially for long-range projections. Such errors will be spotted more reliably if EM-derived wiring diagrams can be compared to a catalog of realistic cell shapes and projection patterns. Our cell-resolved atlas thus provides an anatomical "ground truth," which will be necessary to assemble the complete synapse-scale connectome of the larval zebrafish brain and will contribute to our understanding of brain-wide neuronal dynamics.

\section{$S T A R \star M E T H O D S$}

Detailed methods are provided in the online version of this paper and include the following:

- KEY RESOURCES TABLE

- CONTACT FOR REAGENT AND RESOURCE SHARING

- EXPERIMENTAL MODEL AND SUBJECT DETAILS Zebrafish procedures and lines

- METHOD DETAILS

O PACT tissue clearing

O Immunocytochemistry

$\bigcirc$ Imaging and tracing

o Template generation and image registration

O Registration accuracy

- Quantification of tectal innervation

O Neuron clustering using NBLAST

- Brain segmentation

O Generation of a mesoscale connectome

- QUANTIFICATION AND STATISTICAL ANALYSIS

- DATA AND SOFTWARE AVAILABILITY

\section{SUPPLEMENTAL INFORMATION}

Supplemental Information can be found online at https://doi.org/10.1016/j. neuron.2019.04.034

A video abstract is available at https://doi.org/10.1016/j.neuron.2019.04. 034\#mmc10.

\section{ACKNOWLEDGMENTS}

We thank J. Donovan and M. Al-Kasar for help with the design and implementation of the interactive web portal, C. Bühler for help with tracings, R. Kasper and the MPIN imaging facility for technical support imaging the Gal4 lines, K. Slanchev and S. Steinhäuser for help with fish maintenance and husbandry, M. Wullimann and $\mathrm{O}$. Randlett for feedback on brain annotation, and all members of the Baier lab for helpful discussions. We are grateful to H. Burgess, G. Marquart, Y. Yoshihara, N. Miyasaka, and K. Kawakami for sharing data and fish lines. This study utilized the high-performance computational capabilities of the Linux cluster at the Max Planck Computing and Data Facility (MPCDF, https://www.mpcdf.mpg.de/). Funding was provided by the Max Planck Society and the DFG (SFB 870, Assembly and Function of Neuronal Circuits).

\section{AUTHOR CONTRIBUTIONS}

M.K. and H.B. conceived the experiments and wrote the paper. E.L. performed the experiments. M.K. performed analysis. N.M. wrote the code for the online platform and analysis tools for the single-neuron database. D.F., M.A.F., M.D.M., F.K., and A.K. helped verify the successful alignment of single-neuron tracings.

\section{DECLARATION OF INTERESTS}

The authors declare no competing interests.

Received: September 18, 2018

Revised: April 10, 2019

Accepted: April 23, 2019

Published: May 27, 2019

\section{REFERENCES}

Ahrens, M.B., Orger, M.B., Robson, D.N., Li, J.M., and Keller, P.J. (2013) Whole-brain functional imaging at cellular resolution using light-sheet microscopy. Nat. Methods 10, 413-420.

Akerboom, J., Chen, T.-W., Wardill, T.J., Tian, L., Marvin, J.S., Mutlu, S. Calderón, N.C., Esposti, F., Borghuis, B.G., Sun, X.R., et al. (2012) Optimization of a GCaMP calcium indicator for neural activity imaging. J. Neurosci. 32, 13819-13840.

Akram, M.A., Nanda, S., Maraver, P., Armañanzas, R., and Ascoli, G.A. (2018) An open repository for single-cell reconstructions of the brain forest. Sci. Data 5,180006

Alivisatos, A.P., Chun, M., Church, G.M., Greenspan, R.J., Roukes, M.L., and Yuste, R. (2012). The brain activity map project and the challenge of functional connectomics. Neuron 74, 970-974.

Amo, R., Aizawa, H., Takahoko, M., Kobayashi, M., Takahashi, R., Aoki, T., and Okamoto, H. (2010). Identification of the zebrafish ventral habenula as a homolog of the mammalian lateral habenula. J. Neurosci. 30, 1566-1574.

Asakawa, K., Suster, M.L., Mizusawa, K., Nagayoshi, S., Kotani, T., Urasaki, A., Kishimoto, Y., Hibi, M., and Kawakami, K. (2008). Genetic dissection of neural circuits by Tol2 transposon-mediated Gal4 gene and enhancer trapping in zebrafish. Proc. Natl. Acad. Sci. USA 105, 1255-1260.

Ascoli, G.A., Donohue, D.E., and Halavi, M. (2007). NeuroMorpho.Org: a central resource for neuronal morphologies. J. Neurosci. 27, 9247-9251.

Avants, B.B., Epstein, C.L., Grossman, M., and Gee, J.C. (2008). Symmetric diffeomorphic image registration with cross-correlation: Evaluating automated labeling of elderly and neurodegenerative brain. Med. Image Anal 12, 26-41.

Avants, B.B., Yushkevich, P., Pluta, J., Minkoff, D., Korczykowski, M., Detre, J., and Gee, J.C. (2010). The optimal template effect in hippocampus studies of diseased populations. Neuroimage 49, 2457-2466.

Bae, Y.-K., Kani, S., Shimizu, T., Tanabe, K., Nojima, H., Kimura, Y. Higashijima, S., and Hibi, M. (2009). Anatomy of zebrafish cerebellum and screen for mutations affecting its development. Dev. Biol. 330, 406-426.

Baier, H., and Scott, E.K. (2009). Genetic and optical targeting of neural circuits and behavior-zebrafish in the spotlight. Curr. Opin. Neurobiol. 19, 553-560.

Baier, H., Klostermann, S., Trowe, T., Karlstrom, R.O., Nüsslein-Volhard, C., and Bonhoeffer, F. (1996). Genetic dissection of the retinotectal projection. Development 123, 415-425.

Barker, A.J., and Baier, H. (2015). Sensorimotor decision making in the zebrafish tectum. Curr. Biol. 25, 2804-2814.

Basso, M.A., and May, P.J. (2017). Circuits for Action and Cognition: A View from the Superior Colliculus. Annu. Rev. Vis. Sci. 3, 197-226. 
Bauer, A.Q., Kraft, A.W., Baxter, G.A., Wright, P.W., Reisman, M.D., Bice, A.R., Park, J.J., Bruchas, M.R., Snyder, A.Z., Lee, J.M., and Culver, J.P. (2018). Effective Connectivity Measured Using Optogenetically Evoked Hemodynamic Signals Exhibits Topography Distinct from Resting State Functional Connectivity in the Mouse. Cereb. Cortex 28, 370-386.

Betzel, R.F., and Bassett, D.S. (2018). Specificity and robustness of long-distance connections in weighted, interareal connectomes. Proc. Natl. Acad. Sci. USA 115, E4880-E4889.

Bianco, I.H., Ma, L.-H., Schoppik, D., Robson, D.N., Orger, M.B., Beck, J.C., Li, J.M., Schier, A.F., Engert, F., and Baker, R. (2012). The tangential nucleus controls a gravito-inertial vestibulo-ocular reflex. Curr. Biol. 22, 1285-1295.

Bohland, J.W., Wu, C., Barbas, H., Bokil, H., Bota, M., Breiter, H.C., Cline, H.T., Doyle, J.C., Freed, P.J., Greenspan, R.J., et al. (2009). A proposal for a coordinated effort for the determination of brainwide neuroanatomical connectivity in model organisms at a mesoscopic scale. PLoS Comput. Biol. 5, e1000334.

Bullmore, E., and Sporns, O. (2012). The economy of brain network organization. Nat. Rev. Neurosci. 13, 336-349.

Cannon, R.C., Turner, D.A., Pyapali, G.K., and Wheal, H.V. (1998). An on-line archive of reconstructed hippocampal neurons. J. Neurosci. Methods 84, 49-54.

Chiang, A.S., Lin, C.Y., Chuang, C.C., Chang, H.M., Hsieh, C.H., Yeh, C.W., Shih, C.T., Wu, J.J., Wang, G.T., Chen, Y.C., et al. (2011). Three-dimensional reconstruction of brain-wide wiring networks in Drosophila at single-cell resolution. Curr. Biol. 21, 1-11.

Costa, M., Manton, J.D., Ostrovsky, A.D., Prohaska, S., and Jefferis, G.S.X.E. (2016). NBLAST: Rapid, Sensitive Comparison of Neuronal Structure and Construction of Neuron Family Databases. Neuron 91, 293-311.

Dal Maschio, M., Donovan, J.C., Helmbrecht, T.O., and Baier, H. (2017). Linking Neurons to Network Function and Behavior by Two-Photon Holographic Optogenetics and Volumetric Imaging. Neuron 94, 774-789.

Delignette-Muller, M.L., and Dutang, C. (2015). fitdistrplus : An R package for fitting distributions. J. Stat. Softw 64, 1-34.

Denk, W., and Horstmann, H. (2004). Serial block-face scanning electron microscopy to reconstruct three-dimensional tissue nanostructure. PLoS Biol. 2, е329.

Epskamp, S., Cramer, A.O.J., Waldorp, L.J., Schmittmann, V.D., and Borsboom, D. (2012). qgraph: Network visualizations of relationships in psychometric data. J. Stat. Softw 48, 1-18.

Ercsey-Ravasz, M., Markov, N.T., Lamy, C., Van Essen, D.C., Knoblauch, K., Toroczkai, Z., and Kennedy, H. (2013). A predictive network model of cerebral cortical connectivity based on a distance rule. Neuron 80, 184-197.

Fame, R.M., Brajon, C., and Ghysen, A. (2006). Second-order projection from the posterior lateral line in the early zebrafish brain. Neural Dev. 1, 4.

Fedorov, A., Beichel, R., Kalpathy-Cramer, J., Finet, J., Fillion-Robin, J.-C., Pujol, S., Bauer, C., Jennings, D., Fennessy, F., Sonka, M., et al. (2012). 3D Slicer as an image computing platform for the Quantitative Imaging Network. Magn. Reson. Imaging 30, 1323-1341.

Feng, L., Zhao, T., and Kim, J. (2015). neuTube 1.0: A New Design for Efficient Neuron Reconstruction Software Based on the SWC Format. eNeuro. Published online January 2, 2015. https://doi.org/10.1523/ENEURO.004914.2014.

Filosa, A., Barker, A.J., Dal Maschio, M., and Baier, H. (2016). Feeding State Modulates Behavioral Choice and Processing of Prey Stimuli in the Zebrafish Tectum. Neuron 90, 596-608.

Förster, D., Arnold-Ammer, I., Laurell, E., Barker, A.J., Fernandes, A.M., Finger-Baier, K., Filosa, A., Helmbrecht, T.O., Kölsch, Y., Kühn, E., et al. (2017). Genetic targeting and anatomical registration of neuronal populations in the zebrafish brain with a new set of BAC transgenic tools. Sci. Rep. 7, 5230.

Freeman, J., Vladimirov, N., Kawashima, T., Mu, Y., Sofroniew, N.J., Bennett, D.V., Rosen, J., Yang, C.T., Looger, L.L., and Ahrens, M.B. (2014). Mapping brain activity at scale with cluster computing. Nat. Methods 11, 941-950.
Friedrich, R.W., Genoud, C., and Wanner, A.A. (2013). Analyzing the structure and function of neuronal circuits in zebrafish. Front. Neural Circuits 7, 71.

Hawrylycz, M., Baldock, R.A., Burger, A., Hashikawa, T., Johnson, G.A., Martone, M., Ng, L., Lau, C., Larson, S.D., Nissanov, J., et al. (2011). Digital atlasing and standardization in the mouse brain. PLoS Comput. Biol. 7 , e1001065.

Heap, L., Goh, C.C., Kassahn, K.S., and Scott, E.K. (2013). Cerebellar output in zebrafish: An analysis of spatial patterns and topography in eurydendroid cell projections. Front. Neural Circuits 7, 53.

Heap, L.A.L., Vanwalleghem, G., Thompson, A.W., Favre-Bulle, I.A., and Scott, E.K. (2018). Luminance Changes Drive Directional Startle through a Thalamic Pathway. Neuron 99, 293-301.

Helmbrecht, T.O., Dal Maschio, M., Donovan, J.C., Koutsouli, S., and Baier, H. (2018). Topography of a Visuomotor Transformation. Neuron 100, 1429-1445.

Hildebrand, D.G.C., Cicconet, M., Torres, R.M., Choi, W., Quan, T.M., Moon, J., Wetzel, A.W., Scott Champion, A., Graham, B.J., Randlett, O., et al. (2017). Whole-brain serial-section electron microscopy in larval zebrafish. Nature 545, 345-349.

Horstick, E.J., Jordan, D.C., Bergeron, S.A., Tabor, K.M., Serpe, M., Feldman, B., and Burgess, H.A. (2015). Increased functional protein expression using nucleotide sequence features enriched in highly expressed genes in zebrafish. Nucleic Acids Res. 43, e48-e48.

Jefferis, G.S.X.E., Potter, C.J., Chan, A.M., Marin, E.C., Rohlfing, T., Maurer, C.R., Jr., and Luo, L. (2007). Comprehensive maps of Drosophila higher olfactory centers: spatially segregated fruit and pheromone representation. Cell 128, 1187-1203.

Kinkhabwala, A., Riley, M., Koyama, M., Monen, J., Satou, C., Kimura, Y., Higashijima, S., and Fetcho, J. (2011). A structural and functional ground plan for neurons in the hindbrain of zebrafish. Proc. Natl. Acad. Sci. USA 108, 1164-1169.

Knogler, L.D., Markov, D.A., Dragomir, E.I., Štih, V., and Portugues, R. (2017). Sensorimotor Representations in Cerebellar Granule Cells in Larval Zebrafish Are Dense, Spatially Organized, and Non-temporally Patterned. Curr. Biol. 27, 1288-1302.

Kramer, A., Wu, Y., Baier, H., and Kubo, F. (2019). Neuronal architecture of a visual center that processes optic flow. Neuron 103. Published online May 27, 2019. https://doi.org/10.1016/j.neuron.2019.04.018.

Kubo, F., Hablitzel, B., Dal Maschio, M., Driever, W., Baier, H., and Arrenberg, A.B. (2014). Functional architecture of an optic flow-responsive area that drives horizontal eye movements in zebrafish. Neuron 81, 1344-1359.

Lee, Y.-H., Lin, Y.-N., Chuang, C.-C., and Lo, C.-C. (2014). SPIN: a method of skeleton-based polarity identification for neurons. Neuroinformatics 12 , 487-507.

Li, L., Feng, X., Zhou, Z., Zhang, H., Shi, Q., Lei, Z., Shen, P., Yang, Q., Zhao, B., Chen, S., et al. (2018). Stress Accelerates Defensive Responses to Looming in Mice and Involves a Locus Coeruleus-Superior Colliculus Projection. Curr. Biol. 28, 859-871.

Liao, J.C., and Haehnel, M. (2012). Physiology of afferent neurons in larval zebrafish provides a functional framework for lateral line somatotopy. J. Neurophysiol. 107, 2615-2623.

Manni, E., and Petrosini, L. (2004). A century of cerebellar somatotopy: a debated representation. Nat. Rev. Neurosci. 5, 241-249.

Manton, J.D., Ostrovsky, A.D., Goetz, L., Costa, M., Rohlfing, T., and Jefferis, G.S.X.E. (2014). Combining genome-scale Drosophila 3D neuroanatomical data by bridging template brains. bioRxiv. https://doi.org/10.1101/006353.

Marquart, G.D., Tabor, K.M., Brown, M., Strykowski, J.L., Varshney, G.K., LaFave, M.C., Mueller, T., Burgess, S.M., Higashijima, S., and Burgess, H.A. (2015). A 3D Searchable Database of Transgenic Zebrafish Gal4 and Cre Lines for Functional Neuroanatomy Studies. Front. Neural Circuits 9, 78.

Marquart, G.D., Tabor, K.M., Horstick, E.J., Brown, M., Geoca, A.K., Polys, N.F., Nogare, D.D., and Burgess, H.A. (2017). High-precision registration between zebrafish brain atlases using symmetric diffeomorphic normalization. Gigascience 6, 1-15. 
Matsui, H., Namikawa, K., Babaryka, A., and Köster, R.W. (2014). Functional regionalization of the teleost cerebellum analyzed in vivo. Proc. Natl. Acad. Sci. USA 111, 11846-11851.

McCormick, C.A. (1989). Central Lateral Line Mechanosensory Pathways in Bony Fish. In The Mechanosensory Lateral Line, S. Coombs, P. Görner, and H. Münz, eds. (Springer), pp. 341-364.

McKenna, A., Findlay, G.M., Gagnon, J.A., Horwitz, M.S., Schier, A.F., and Shendure, J. (2016). Whole-organism lineage tracing by combinatorial and cumulative genome editing. Science 353, aaf7907.

Meek, J. (1983). Functional anatomy of the tectum mesencephali of the goldfish. An explorative analysis of the functional implications of the laminar structural organization of the tectum. Brain Res. 287, 247-297.

Mitra, P.P. (2014). The circuit architecture of whole brains at the mesoscopic scale. Neuron 83, 1273-1283.

Miyasaka, N., Arganda-Carreras, I., Wakisaka, N., Masuda, M., Sümbül, U., Seung, H.S., and Yoshihara, Y. (2014). Olfactory projectome in the zebrafish forebrain revealed by genetic single-neuron labelling. Nat. Commun. 5, 3639.

Mueller, T., and Wullimann, M. (2005). Atlas of Early Zebrafish Brain Development, 2nd Edition | Dr. Thomas Mueller, Mario Wullimann | ISBN 9780124186699

Naumann, E.A., Fitzgerald, J.E., Dunn, T.W., Rihel, J., Sompolinsky, H., and Engert, F. (2016). From Whole-Brain Data to Functional Circuit Models: The Zebrafish Optomotor Response. Cell 167, 947-960.

Oh, S.W., Harris, J.A., Ng, L., Winslow, B., Cain, N., Mihalas, S., Wang, Q., Lau, C., Kuan, L., Henry, A.M., et al. (2014). A mesoscale connectome of the mouse brain. Nature 508, 207-214.

Ohyama, T., Schneider-Mizell, C.M., Fetter, R.D., Aleman, J.V., Franconville, R., Rivera-Alba, M., Mensh, B.D., Branson, K.M., Simpson, J.H., Truman, J.W., et al. (2015). A multilevel multimodal circuit enhances action selection in Drosophila. Nature 520, 633-639.

Orger, M.B., and de Polavieja, G.G. (2017). Zebrafish Behavior: Opportunities and Challenges. Annu. Rev. Neurosci. 40, 125-147.

Otsuna, H., Hutcheson, D.A., Duncan, R.N., McPherson, A.D., Scoresby, A.N., Gaynes, B.F., Tong, Z., Fujimoto, E., Kwan, K.M., Chien, C.B., et al. (2015). High-resolution analysis of central nervous system expression patterns in zebrafish Gal4 enhancer-trap lines. Dev. Dyn 244, 785-796.

Pandey, S., Shekhar, K., Regev, A., and Schier, A.F. (2018). Comprehensive Identification and Spatial Mapping of Habenular Neuronal Types Using Single-Cell RNA-Seq. Curr. Biol. 28, 1052-1065.e7.

Panzeri, S., Harvey, C.D., Piasini, E., Latham, P.E., and Fellin, T. (2017). Cracking the Neural Code for Sensory Perception by Combining Statistics, Intervention, and Behavior. Neuron 93, 491-507.

Pérez-Pérez, M.P., Luque, M.A., Herrero, L., Núñez-Abades, P.A., and Torres, B. (2003). Afferent connectivity to different functional zones of the optic tectum in goldfish. Vis. Neurosci. 20, 397-410.

Portugues, R., Feierstein, C.E., Engert, F., and Orger, M.B. (2014). Whole-brain activity maps reveal stereotyped, distributed networks for visuomotor behavior. Neuron 81, 1328-1343.

Raj, B., Wagner, D.E., McKenna, A., Pandey, S., Klein, A.M., Shendure, J., Gagnon, J.A., and Schier, A.F. (2018). Simultaneous single-cell profiling of lineages and cell types in the vertebrate brain. Nat. Biotechnol. 36, 442-450.

Randlett, O., Wee, C.L., Naumann, E.A., Nnaemeka, O., Schoppik, D., Fitzgerald, J.E., Portugues, R., Lacoste, A.M., Riegler, C., Engert, F., and Schier, A.F. (2015). Whole-brain activity mapping onto a zebrafish brain atlas. Nat. Methods 12, 1039-1046.

Robles, E., Filosa, A., and Baier, H. (2013). Precise lamination of retinal axons generates multiple parallel input pathways in the tectum. J. Neurosci. 33, 5027-5039.
Robles, E., Laurell, E., and Baier, H. (2014). The retinal projectome reveals brain-area-specific visual representations generated by ganglion cell diversity. Curr. Biol. 24, 2085-2096.

Roeser, T., and Baier, H. (2003). Visuomotor behaviors in larval zebrafish after GFP-guided laser ablation of the optic tectum. J. Neurosci. 23, 3726-3734.

Satou, C., Kimura, Y., Hirata, H., Suster, M.L., Kawakami, K., and Higashijima, S. (2013). Transgenic tools to characterize neuronal properties of discrete populations of zebrafish neurons. Development 140, 3927-3931.

Schindelin, J., Arganda-Carreras, I., Frise, E., Kaynig, V., Longair, M., Pietzsch, T., Preibisch, S., Rueden, C., Saalfeld, S., Schmid, B., et al. (2012). Fiji: an open-source platform for biological-image analysis. Nat. Methods 9, 676-682. Scott, E.K., Mason, L., Arrenberg, A.B., Ziv, L., Gosse, N.J., Xiao, T., Chi, N.C. Asakawa, K., Kawakami, K., and Baier, H. (2007). Targeting neural circuitry in zebrafish using GAL4 enhancer trapping. Nat. Methods 4, 323-326.

Semmelhack, J.L., Donovan, J.C., Thiele, T.R., Kuehn, E., Laurell, E., and Baier, H. (2014). A dedicated visual pathway for prey detection in larval zebrafish. eLife 3, 4878.

Shih, C.T., Sporns, O., Yuan, S.L., Su, T.S., Lin, Y.J., Chuang, C.C., Wang, T.Y., Lo, C.C., Greenspan, R.J., and Chiang, A.S. (2015). Connectomicsbased analysis of information flow in the Drosophila brain. Curr. Biol. 25 1249-1258.

Sporns, O., Tononi, G., and Kötter, R. (2005). The human connectome: A structural description of the human brain. PLoS Comput. Biol. 1, e42.

Springer, A.D., and Mednick, A.S. (1985). A quantitative study of the relative contribution of different retinal sectors to the innervation of various thalamic and pretectal nuclei in goldfish. J. Comp. Neurol. 242, 369-380.

Stuermer, C.A. (1988). Retinotopic organization of the developing retinotectal projection in the zebrafish embryo. J. Neurosci. 8, 4513-4530.

Thompson, A.W., Vanwalleghem, G.C., Heap, L.A., and Scott, E.K. (2016). Functional Profiles of Visual-, Auditory-, and Water Flow-Responsive Neurons in the Zebrafish Tectum. Curr. Biol. 26, 743-754.

Treweek, J.B., Chan, K.Y., Flytzanis, N.C., Yang, B., Deverman, B.E. Greenbaum, A., Lignell, A., Xiao, C., Cai, L., Ladinsky, M.S., et al. (2015). Whole-body tissue stabilization and selective extractions via tissue-hydrogel hybrids for high-resolution intact circuit mapping and phenotyping. Nat Protoc. 10, 1860-1896.

Turner, K.J., Hawkins, T.A., Yáñez, J., Anadón, R., Wilson, S.W., and Folgueira M. (2016). Afferent Connectivity of the Zebrafish Habenulae. Front. Neura Circuits 10, 30

Vanwalleghem, G.C., Ahrens, M.B., and Scott, E.K. (2018). Integrative wholebrain neuroscience in larval zebrafish. Curr. Opin. Neurobiol. 50, 136-145.

Varshney, L.R., Chen, B.L., Paniagua, E., Hall, D.H., and Chklovskii, D.B. (2011). Structural properties of the Caenorhabditis elegans neuronal network. PLoS Comput. Biol. 7, e1001066.

Wan, Y., Otsuna, H., Chien, C.B., and Hansen, C. (2012). FluoRender: An application of $2 \mathrm{D}$ image space methods for $3 \mathrm{D}$ and $4 \mathrm{D}$ confocal microscopy data visualization in neurobiology research. 2012 IEEE Pacific Visualization Symposium (IEEE), pp. 201-208.

Xiao, T., and Baier, H. (2007). Lamina-specific axonal projections in the zebrafish tectum require the type IV collagen Dragnet. Nat. Neurosci. 10, 1529-1537.

Xiao, T., Roeser, T., Staub, W., and Baier, H. (2005). A GFP-based genetic screen reveals mutations that disrupt the architecture of the zebrafish retinotectal projection. Development 132, 2955-2967.

Zheng, Z., Lauritzen, J.S., Perlman, E., Robinson, C.G., Nichols, M., Milkie, D., Torrens, O., Price, J., Fisher, C.B., Sharifi, N., et al. (2018). A Complete Electron Microscopy Volume of the Brain of Adult Drosophila melanogaster. Cell 174, 730-743. 


\section{STAR $\star$ METHODS}

\section{KEY RESOURCES TABLE}

\begin{tabular}{|c|c|c|}
\hline REAGENT or RESOURCE & SOURCE & IDENTIFIER \\
\hline \multicolumn{3}{|l|}{ Deposited Data } \\
\hline Single-neuron tracings & This paper & https://fishatlas.neuro.mpg.de/neurons/main_page \\
\hline Gal4-expression pattern & This paper & https://fishatlas.neuro.mpg.de/lines \\
\hline \multicolumn{3}{|l|}{ Antibodies } \\
\hline Chicken anti-GFP & Invitrogen & A10262, RRID: AB_2534023 \\
\hline Rabbit anti-ERK1/2 & Cell Signaling Technology & 9102, RRID: AB_330744 \\
\hline Mouse anti-HuC/D & Invitrogen & A21271, RRID:AB_221448 \\
\hline Rabbit anti-Synapsin 1/2 & Synaptic Systems & 106002, RRID:AB_887804 \\
\hline \multicolumn{3}{|l|}{ Chemicals, Peptides, and Recombinant Proteins } \\
\hline Photoinitiator & Wako Chemicals & VA-044 \\
\hline \multicolumn{3}{|l|}{ Experimental Models: Organisms/Strains } \\
\hline $\begin{array}{l}\text { Zebrafish Tg(brn3c:GAL4, UAS:gap43-GFP)s318t } \\
\text { (BGUG) }\end{array}$ & Xiao and Baier, 2007 & ZFIN ID: ZDB-ALT-070423-6 \\
\hline Zebrafish Tg(Et(e1b:GAL4)s1101t) & Scott et al., 2007 & ZFIN ID: ZDB-ALT-070716-2 \\
\hline Zebrafish Tg(elav/3:Gal4)zf349 & Akerboom et al., 2012 & ZFIN ID: ZDB-ALT-121024-4 \\
\hline $\begin{array}{l}\text { Zebrafish Tg(14xUAS:mCherry)s1984t, TgBAC } \\
\text { (slc17a6b:Gal4FF) zK145P24 }\end{array}$ & $\begin{array}{l}\text { Heap et al., 2013; } \\
\text { Satou et al., } 2013\end{array}$ & $\begin{array}{l}\text { ZFIN ID: ZDB-ALT-130702-1; } \\
\text { ZFIN ID: ZDB-ALT-131127-1 }\end{array}$ \\
\hline $\begin{array}{l}\text { Zebrafish TgBAC(vglut2a[s/c17a6b]:loxP-DsRed- } \\
\text { loxP-GFP)nns14 }\end{array}$ & Satou et al., 2013 & ZFIN ID: ZDB-ALT-110413-5 \\
\hline Zebrafish Tg(UAS-E1b:NfsB-mCherry)c264 & Otsuna et al., 2015 & ZFIN ID: ZDB-ALT-070316-1 \\
\hline Zebrafish Tg(elav/3:lyn-tagRFP)mpn404 & Dal Maschio et al., 2017 & ZFIN ID: ZDB-ALT-170731-38 \\
\hline Zebrafish Tg(elavl3:n/sGCaMP6s)mpn400 & Dal Maschio et al., 2017 & ZFIN ID: ZDB-ALT-170731-37 \\
\hline Zebrafish Tg(actb2:IoxP-eGFP-loxP-lynTagRFPT)y272 & Horstick et al., 2015 & ZFIN ID: ZDB-ALT-150721-8 \\
\hline Zebrafish Tg(elav/3:Hsa.H2B-GCaMP6s)jf5 & Freeman et al., 2014 & ZFIN ID: ZDB-ALT-141023-2 \\
\hline Zebrafish Tg(elav/3:GCaMP5G)a4598 & Ahrens et al., 2013 & ZFIN ID: ZDB-ALT-130924-1 \\
\hline Zebrafish Tg(sst3: Gal4-VP16)mpn219 & Förster et al., 2017 & ZFIN ID: ZDB-ALT-170908-20 \\
\hline Zebrafish Tg(galn:Gal4-VP16)mpn213 & Förster et al., 2017 & ZFIN ID: ZDB-ALT-170908-13 \\
\hline Zebrafish Tg(gad1b/GAD67:Gal4-VP16)mpn155 & Förster et al., 2017 & ZFIN ID: ZDB-ALT-160726-5 \\
\hline Zebrafish Tg(chata:Gal4-VP16)mpn202 & Förster et al., 2017 & ZFIN ID: ZDB-ALT-170908-4 \\
\hline Zebrafish Tg(drd2a:Gal4-VP16)mpn211 & Förster et al., 2017 & ZFIN ID: ZDB-ALT-170908-9 \\
\hline Zebrafish $\operatorname{Tg}(E t(-1.5 h s p 70 l: G a l 4-V P 16) s 1013 t)$ & Scott et al., 2007 & ZFIN ID: ZDB-ALT-070420-14 \\
\hline Zebrafish $\operatorname{Tg}(E t(-0.6 h s p 70 l: G a l 4-V P 16) s 1020 t)$ & Scott et al., 2007 & ZFIN ID: ZDB-ALT-070420-21 \\
\hline Zebrafish $\operatorname{Tg}(E t(f o s: G a l 4-V P 16) s 1168 t)$ & Scott et al., 2007 & ZFIN ID: ZDB-ALT-080327-136 \\
\hline Zebrafish $\operatorname{Tg}(E t(-0.6 h s p 70 l: G a l 4-V P 16) s 1171 t)$ & Scott et al., 2007 & ZFIN ID: ZDB-ALT-080327-139 \\
\hline Zebrafish $\operatorname{Tg}(E t(f o s: G a l 4-V P 16) s 1113 t)$ & Scott et al., 2007 & ZFIN ID: ZDB-ALT-080327-86 \\
\hline \multicolumn{3}{|l|}{ Software and Algorithms } \\
\hline Advanced Normalization Tools (ANTs) & Avants et al., 2008 & http://stnava.github.io/ANTs/ \\
\hline NeuTube & Feng et al., 2015 & https://www.neutracing.com/ \\
\hline Fiji & Schindelin et al., 2012 & https://fiji.sc/ \\
\hline FluoRender & Wan et al., 2012 & http://www.sci.utah.edu/software/fluorender.html \\
\hline 3DSlicer & Fedorov et al., 2012 & https://www.slicer.org/ \\
\hline Python 2.7 & Python.org & https://www.python.org/ \\
\hline $\mathrm{R}$ & The R project & https://cran.r-project.org/ \\
\hline
\end{tabular}




\begin{tabular}{|c|c|c|}
\hline Continued & & \\
\hline REAGENT or RESOURCE & SOURCE & IDENTIFIER \\
\hline $\mathrm{R}$ nat package & Jefferis et al., 2007 & $\begin{array}{l}\text { https://cran.r-project.org/web/packages/nat/ } \\
\text { index.html }\end{array}$ \\
\hline $\mathrm{R}$ nat.nblast package & Costa et al., 2016 & $\begin{array}{l}\text { https://cran.r-project.org/web/packages/nat. } \\
\text { nblast/index.html }\end{array}$ \\
\hline $\mathrm{R}$ fitdistrplus package & $\begin{array}{l}\text { Delignette-Muller and } \\
\text { Dutang, } 2015\end{array}$ & $\begin{array}{l}\text { https://cran.r-project.org/web/packages/ } \\
\text { fitdistrplus/index.html }\end{array}$ \\
\hline R iGraph package & & https://igraph.org/ \\
\hline R rgl package & & $\begin{array}{l}\text { https://cran.r-project.org/web/packages/rgl/ } \\
\text { index.html }\end{array}$ \\
\hline R qgraph package & Epskamp et al., 2012 & https://cran.r-project.org/web/packages/qgraph/ \\
\hline $\mathrm{R}$ braingraph package & & https://cran.r-project.org/web/packages/qgraph/ \\
\hline
\end{tabular}

\section{CONTACT FOR REAGENT AND RESOURCE SHARING}

Further information and requests for resources and reagents should be directed to and will be fulfilled by the Lead Contact, Herwig Baier (hbaier@neuro.mpg.de).

\section{EXPERIMENTAL MODEL AND SUBJECT DETAILS}

For all experiments in this study we used 5-7 days post fertilization (d.p.f.) larvae. At this stage, the sex of the larvae is not defined. All animal procedures conformed to the institutional guidelines set by the Max Planck Society and were carried out in accordance with an animal protocol approved by the regional government (Regierung von Oberbayern).

\section{Zebrafish procedures and lines}

Fish were raised at $28^{\circ} \mathrm{C}$ on a $14 \mathrm{~h}$ light/10h dark cycle in Danieau's solution $(17 \mathrm{mM} \mathrm{NaCl}, 2 \mathrm{mM} \mathrm{KCl,} 0.12 \mathrm{mM} \mathrm{MgSO}, 1.8 \mathrm{mM}$ $\mathrm{Ca}\left(\mathrm{NO}_{3}\right)_{2}, 1.5 \mathrm{mM}$ HEPES). Pigmented larvae destined to be imaged were kept in Danieau's solution containing $0.003 \%$ 1-phenyl-2-thiourea from the age of $24 \mathrm{hpf}$ (hours post-fertilization) to inhibit melanogenesis. For single neuron labeling, the transgenic fish line: $T g(b r n 3 c: G A L 4$, UAS:gap43-GFP)s318t (BGUG) was crossed to either transgenic fishline Tg(elav/3:Gal4)zf349 or $\operatorname{Tg}$ (Et(e1b:GAL4)s1101t. These larvae were subsequently PACT cleared, immunostained and imaged as fixed samples (see below). To establish a segmented brain atlas as well as to align the expression patterns of various transgenic fish lines to our standard brain, Tg(elav/3:Iyn-tagRFP)mpn404 and Tg(actb2:loxP-eGFP-loxP-lynTagRFPT)y272 were crossed to transgenic Gal4 lines from https:// fishatlas.neuro.mpg.de/zebrafishatlas/ and the following transgenic fish lines: $T$ g(elavl3:n/sGCaMP6s)mpn400, Tg(elavl3:Hsa.H2BGCaMP6s) and Tg(elav/3:GCaMP5G)a4598. These larvae were imaged live (see below).

\section{METHOD DETAILS}

\section{PACT tissue clearing}

At $6 \mathrm{dpf}$, larvae were anesthetized in a lethal dose of tricaine for 5 minutes and thereafter transferred into $10 \mathrm{~mL}$ ice-cold PACT hydrogel monomer solution $\mathrm{A}_{4} \mathrm{P}_{4}$ (4\% acrylamide and $4 \%$ paraformaldehyde in PBS) supplemented with $0.25 \% \mathrm{VA}-044$, and kept at $4{ }^{\circ} \mathrm{C}$ over night. For degassing, samples were placed with an open lid in a desiccation chamber filled with dry ice. Oxygen was removed via a vacuum pump and the desiccation chamber was thereafter transferred to a $55-60^{\circ} \mathrm{C}$ water bath so the dry ice would melt and fill the chamber with $\mathrm{CO}_{2}$. After all oxygen was replaced by $\mathrm{CO}_{2}$, the tube lid was closed, avoiding any exposure to oxygen, and the tubes were removed from the desiccation chamber and placed on a shaker at $37^{\circ} \mathrm{C}$ for two hours to facilitate the polymerization of the hydrogel. Samples were transferred from the hydrogel solution and put into $50 \mathrm{~mL}$ canonical tubes containing clearing solution (4\% SDS and $200 \mathrm{mM}$ boric acid in $\mathrm{dH}_{2} \mathrm{O}$ ) and kept at $37^{\circ} \mathrm{C}$ for 5-6 days under gentle shaking. During the clearing step, the solution was changed every other day. Once clearing was complete, samples were washed 3 times for 10 minutes in PBT and thereafter kept in PBT at $4^{\circ} \mathrm{C}$ over night or longer.

\section{Immunocytochemistry}

Samples were incubated in $150 \mathrm{mM}$ Tris- $\mathrm{HCl}, \mathrm{pH}$ 9, for $5 \mathrm{~min}$ at room temperature followed by 15 min at $63^{\circ} \mathrm{C}$ for antigen retrieval. Larvae were washed again in PBT prior to a 45 min digest with Trypsin EDTA (1x Trypsin-EDTA Solution, Sigma, diluted 1:20) on ice. PBT-washed larvae were blocked in 5\% goat serum, 1\% BSA, and 1\% DMSO in PBT. Primary antibodies were added in blocking 
solution for 5 days. Secondary antibodies were Alexa-conjugates (Invitrogen) and added 1:300 in PBT for 3-4 days at $4{ }^{\circ} \mathrm{C}$. Washed samples were postfixed for $45 \mathrm{~min}$ in $4 \%$ paraformaldehyde at room temperature, rinsed, and thereafter transferred to $87 \% \mathrm{glycerol}$.

\section{Imaging and tracing}

For live imaging, 6-7 dpf larvae were anesthetized in $0.016 \%$ tricaine and embedded in $2 \%$ low-melting-point agarose. Imaging was performed on a Zeiss LSM-780, a LSM-700, or a Leica SP8 confocal microscope, using a $20 \times / 1.0$ NA water-dipping objective. For imaging fixed samples, larvae were embedded in $87 \%$ glycerol, and imaging was performed at a Zeiss LSM-780 using a $25 \times / 0.8 \mathrm{NA}$ multi-immersion objective. In both cases, whole-brain images were acquired by tiling the brain into two individual image stacks along the anterior posterior axis and subsequently stitched, using either ZEN software (black edition, v8.0; Zeiss) or LAS X on the SP8. Images were corrected for fluorescence attenuation in the $z$-dimension using the brightness correction function in the respective software. Samples used for single neuron tracing were scanned at a resolution of $0.3 \times 0.3 \times 0.3 \mu \mathrm{m}$ (xyz), while Gal4 expression pattern were scanned with isotropic voxel size of $1 \mu \mathrm{m}$.

Individual neurons were semi-automatically traced using the freeware NeuTube (Feng et al., 2015) and saved as individual swc files (Cannon et al., 1998). In total, we scanned 1278 fish for a total of 1955 tracings (1.53 tracings per fish).

\section{Template generation and image registration}

For the original template we generated an unbiased shape-based average template by using antsMultivariateTemplateConstruction2.sh (Avants et al., 2010), a shell script in the Advanced Normalization Tools (ANTs) software. We chose 12 representative brains stained for the presynaptic marker Synapsin (SYP). This process is iterative and leads to an improvement in template quality with each iteration. A template after a single iteration (Figure 1C1; Figures S1A-S1C) already displays marked improvements compared to each of the individual fish that were used to generate the average brain by cancelling out/removing the deformations introduced by our PACT protocol. However, despite these advantages, the template appears to be blurry and it is hard to discern finer anatomical details. For example, it is very difficult to recognize the neuropil of the torus longitudinalis (TLn; Figure 1C1, inset) or the individual layers of the optic tectum (Figure S1A) as well as individual identifiable cells of the reticulospinal system (e.g., Mauthner cells; Figures S1B and S1C). After four iterations (Figure 1C2; Figures S1D-S1F) the general appearance of the template was markedly improved, as evidenced by the clear emergence of the TLn (Figure 1C2, inset) as well as the individual tectal layers (Figure S1D) and reticulospinal neurons. (Figures S1E and S1F). Additional reference stains were imaged with a second channel containing a marker already present in our common coordinate framework and this marker was used as a bridge to our template space. Brain with single-neuron labeling were aligned to our standard brain using either SYP, HuC/D, or ERK1/2 or a combination thereof using the parameters for fixed samples described in (Marquart et al., 2017) using the antsRegistration function in ANTs. Single neuron tracings were aligned using the antsApplyTransformToPoints function. The quality of alignment is strongly dependent on the original image quality. Furthermore, the image quality can be vastly different between brain regions in an individual fish. To ensure that alignment quality of neurons is high, aligned brains were manually scored by expert analysts and only neurons that innervated regions that were deemed as successfully aligned were used for further analysis.

For alignment of GAL4 pattern imaged live we generated a shape based average templates of the transgenic lines $T g(e l a v / 3: / y n-$ tagRFP)mpn404 and Tg(actb2:loxP-eGFP-loxP-lynTagRFPT)y272 using the procedure described above. Gal4 lines were crossed to one of those references and to either Tg(UAS:Dendra-kras)s1998t, Tg(5xUAS:EGFP) or, Tg(UAS-E1b:NFsB-mCherry) to visualize Gal4 expression patterns. For each line we imaged 3-6 larvae and generated a shape-based average brain. We aligned both the types of images of the individual brains as well as the shape-based averages to our live template brain. We again used the optimal parameters for alignment of live-images as described previously (Marquart et al., 2017). To compare Gal4-expression obtained through live imaging with single-neuron data from fixed samples we generated a bridging registration between live and fixed. We chose four lines (TgBAC(vglut2a[s/c17a6b]:loxP-DsRed-loxP-GFP)nns9, Tg(tph2:nfsB-mCherry)y226, Tg(elav/3:lyn-tagRFP)mpn404, Tg(is/1:GFP)) that displayed similar expression when imaged live or after fixation, clearing, and staining. We used a similar approach to integrate our Gal4 database with the Z-Brain and zebrafish brain browser (ZBB). We chose four lines present in the ZBB database (Tg(elav/3n/smCardinal), TgBAC(gad1b:GFP), Tg(is/1:GFP), TgBAC(vglut2a[s/c17a6b]:IoxP-DsRed-loxP-GFP)nns9) and aligned them to the respective lines in our database (Tg(elav/3:n/sGCaMP6s)mpn400, TgBAC(gad1b:GFP), Tg(is/1:GFP), TgBAC(vglut2a[s/c17a6b]: loxP-DsRed-loxP-GFP)nns9). For incorporation of Z-Brain we made use of the fact that their database has been already incorporated into ZBB and we just applied the transformations to the data present in ZBB.

\section{Registration accuracy}

To measure the registration accuracy, we first identified the various fiducial points in the original unidentified image stacks using the Slicer software and saved their $x, y$, and $z$ coordinates in a text file. Next, we registered those stacks using a reference channel to our standard brain and applied the transformations to coordinates of the fiducial points.

\section{Quantification of tectal innervation}

To measure the relative innervation of individual tectal layers by retinal and non-retinal afferents we measured the total length of all fibers within the tectal neuropil on each side of the brain. Since RGCs all project to the contralateral tectum we mirrored all RGCs to both hemispheres. Ipsi- and contralateral labels in Figure 5B relates to the soma location of non-retinal afferents. Next, we measured 
the fibers length in each tectal layer and divided it by the total length per tectum to calculate the relative contribution of each cell group for the individual layers.

\section{Neuron clustering using NBLAST}

To flip all neurons to one hemisphere of the brain we followed the procedure described in (Manton et al., 2014). Briefly, to create a mirroring registration we chose four representative stacks (TgBAC(gad1b:GFP), TgBAC(vglut2a[s/c17a6b]:IoxP-DsRed-loxP-GFP) $n n s 9), \alpha-H u C / D$, and $\alpha$-SYP), flipped those images along the horizontal axis and registered the original versions of the stacks to the flipped version using ANTs as described previously. We manually determined the midline of the brain and categorized neurons as left or right hemispheric based on soma location. Neurons were flipped to the opposite side of the brain and the mirroring registration was applied using the antsApplyTransformToPoints function. Pairwise NBLAST scores were computed either for all cerebellar efferents or for the entire dataset (the nblast_allbyall function in the nat.nblast package at https://github.com/jefferislab/nat.nblast was used). We used unsupervised hierarchical clustering with Ward's method based on the NBLAST scores (the nhclust function in the nat package at https://github.com/jefferis/nat was used). Visualization was performed using the plot.neuron and plot.surfaces function of the nat package.

\section{Brain segmentation}

To provide a framework for graph theoretical analysis we manually segmented the brain into 36 brain regions using the open source software 3D slicer (https://www.slicer.org/). Neuroanatomical annotation was based primarily on (Mueller and Wullimann, 2005) with the main exception of the individual stripes in the medulla oblongata which was based on (Kinkhabwala et al., 2011). Specific information about the brain regions can be found in Table S1. To aid in determining brain region boundaries which are notoriously hard to determine in larval zebrafish we made use of the large collection of Gal4 lines and expression patterns present in our database. If possible, we determined a brain region based on a single expression pattern (e.g., Is/1 to label cranial nuclei) but more often we used a combination of multiple expression pattern.

\section{Generation of a mesoscale connectome}

For generating an interareal connectivity matrix we split the 36 brain regions into left and right hemisphere, leaving us with 72 brain regions. To determine the connectivity between brain regions we measured which brain regions a neuron innervated. We only considered a brain region to be innervated by a neuron if a neuron had either tips or a soma in it. This was done to prevent false positive assignment of neurons whose fibers only pass through a region. Since our dataset only comprises about $2 \%$ of the total number of brain cells and almost all neurons in the brain are bilaterally symmetric, we decided to double our dataset and mirror all neurons to the contralateral hemisphere. Furthermore, we only considered neurons whose fiber length in a brain region exceeds $10 \mu \mathrm{m}$ to excluded false positive results from neurons that are located at the border of brain regions that gets falsely assigned to a brain region due to alignment inaccuracies. We removed all local interneurons by excluding neurons that can only be assigned to one brain region. We converted this dataset into an adjacency matrix by summing the lengths for all neurons that connect a pair of brain regions the following way:

$\operatorname{Data}[R \times N]$ is a matrix that contains the lengths of all the neurons in each brain region where $(R)$ is the number of brain regions, and $(N)$ is the number of neurons.

$$
\text { Data }=\left[\begin{array}{ccccc}
n_{00} & n_{01} & n_{02} & \ldots & n_{0 N} \\
n_{10} & n_{11} & n_{12} & \ldots & n_{1 N} \\
n_{20} & n_{21} & n_{22} & \ldots & n_{2 N} \\
\ldots & \ldots & \ldots & \ldots & \ldots \\
n_{R 0} & n_{R 1} & n_{R 2} & \ldots & n_{R N}
\end{array}\right]
$$

where Data $[i, j]=n_{i j}$ : neuron's $(j)$ length inside region $(i)$.

$C M[R \times R]$ is the connectivity matrix.

$$
C M=\left[c m_{i j}\right], \text { where } c m_{i j}=\sum_{k=1}^{N}(\operatorname{Data}[i, k]+\operatorname{Data}[j, k]) * \omega
$$

Where:

$$
\omega=\left\{\begin{array}{c}
1, \mid i \neq j \Lambda \text { Data }[i, k] \geq m I \Lambda \text { Data }[j, k] \geq m l \\
0, \mid \text { Otherwise } \\
i, j \in[0, R]
\end{array}\right.
$$

$m l:$ is the minimum accepted length of a neuron inside a brain region $(m l=10 \mu)$ 


$$
C M=\left[\begin{array}{ccccc}
0 & c m_{01} & c m_{02} & \ldots & c m_{0 R} \\
c m_{10} & 0 & c m_{12} & \ldots & c m_{1 R} \\
c m_{20} & c m_{21} & 0 & \ldots & c m_{2 R} \\
\ldots & \ldots & \ldots & \ldots & \ldots \\
c m_{R 0} & c m_{R 1} & c m_{R 2} & \ldots & 0
\end{array}\right]
$$

Since our annotation contains brain regions of various sizes and to remove any bias in connection strengths arising from this we normalized connection strengths by dividing it by the volume of the pair of brain regions connected.

\section{QUANTIFICATION AND STATISTICAL ANALYSIS}

To measure the distance between the aligned fiducial points and the same point identified in the standard brain (Figure $3 \mathrm{~A}$ ) we measured the Euclidean distance using the dist function of the $\mathrm{R}$ base package. For determining the distribution of normalized connection strength (Figure 8A), we used the fitdistrplus package provided by R (https://cran.r-project.org/web/packages/ fitdistrplus/index.html). Distances between brain regions (Figure 8B) were calculated as the Euclidean distance between the center of mass for each pair of brain regions and the correlation between the connection strength was calculated using the cor function in $\mathrm{R}$. To order brain region based on similar connectivity profiles (Figure $8 \mathrm{C}$ ) we performed unsupervised hierarchical clustering using Wards's method.

\section{DATA AND SOFTWARE AVAILABILITY}

All custom software will be made available upon request. Neuron tracings, brain region segmentations, and transformation files are available for download on a special section on our web-portal (https://fishatlas.neuro.mpg.de/neurons/downloads). 